\title{
Higher order dynamic mode decomposition of noisy experimental data: The flow structure of a zero-net-mass-flux jet
}

\author{
Soledad Le Clainche ${ }^{a, *}$, José M. Vega ${ }^{a}$, Julio Soria ${ }^{b, c}$
}

\begin{abstract}
A B S T R A C T
A method is presented to treat complex experimental flow data resulting from PIV. The method is based on an appropriate combination of higher order singular value decomposition (which cleans the data along the temporal dimension and the various space dimensions) and higher order dynamic mode decomposition (HODMD), a recent extension of standard dynamic mode decomposition that treats the data in a sliding window. The performance of the method is tested using experimental data obtained in the near field of a zero-net-mass-flux (ZNMF) jet. The better performance of HODMD is put in evidence making this technique suitable to both, cleaning the experimental noise using a limited number of snapshots and obtaining robust and sufficiently accurate results that elucidate the spatio-temporal structure of the flow. The results show that this ZNMF jet is temporally periodic in the near field, where the flow results from the interaction of a large number harmonics. These harmonics involve large scale spatial flow structures, identified as spatially growing instabilities, which are associated with the flow transition to turbulence in the far field.
\end{abstract}

\section{Introduction}

The deep study and understanding of complex flow behavior, such as transitional or turbulent flows, is a research topic of high interest. The main reason is that these type of flows are not only found in nature, but also cover a wide range of industrial applications. Although these flows have been studied for decades, due to their high complexity, to elucidate their spatio-temporal structure is still considered an open topic in a large quantity of relevant cases. For instance, understanding and controlling the zero-netmass-flux (ZNMF) jet $[5,41]$, namely the pulsatile jet produced through an orifice by periodically oscillating a membrane or a piston in a cavity, requires resolving the various, fairly disparate, spatio-temporal scales that are involved in this flow. On the other hand, this flow is related to some pulsatile jets that are relevant in nature (e.g., jellyfish swimming [12]) and is of interest in connection with synthetic jet actuators [16], which exhibit potential applications in the areas of mixing enhancement [44], heat transfer [29], jet vectoring [37], and active flow control of separation [7].

A good way of understanding complex flows is finding coherence in fluid motions. Thus, in the past, several techniques have been developed and aimed at finding well organized and structured flow patterns. Among these techniques, some of the most popular are based on the statistical analysis of turbulent flows [1], on the decomposition of the second invariant of velocity gradient $[20,19]$, or even into the proper orthogonal decomposition (POD) of the flow, represented as an expansion of the most energetic POD modes [38].

Dynamic mode decomposition (DMD) is a technique that gives an approximation of the Koopman modes [22,31], which make it a suitable candidate to analyze unsteady flow dynamics and flow structures. Schmid [32] introduced the algorithm and encouraged it to be used to study the associated growth rates and frequencies in both linear and non-linear flows.

Focussing on applying DMD to analyze standard particle image velocimetry (PIV) measurements, which are usually performed in a plane, $x y$, the method approximates the instantaneous flow field measurements (snapshots) at $K$ equispaced values in time $(\Delta t)$, where $K$ will be called the temporal dimension. The vectors $\boldsymbol{v}\left(t_{k}\right) \equiv \boldsymbol{v}_{k} \in R e^{l}$ correspond to the snapshots, where $t_{k}=(k-1) \Delta t$ for $k=1, \ldots, K$. The common size of these vectors, $J$, is the spatial dimension, defined in terms of the number of grid-points in $x$ and $y$ where the two in-plane velocity components are measured, as $J=2\left(n_{x} \times n_{y}\right)$. 
DMD gives a Fourier-like expansion in spatial modes in the following way

$\boldsymbol{v}_{k} \simeq \boldsymbol{v}_{k}^{\mathrm{DMD}} \equiv \sum_{m=1}^{M} a_{m} \boldsymbol{u}_{m} \mathrm{e}^{\left(\delta_{m}+\mathrm{i} \omega_{m}\right)(k-1) \Delta t} \quad$ for $k=1, \ldots, K$,

where the number of terms (number of DMD modes), $M$, can be referred to as the spectral complexity, $\boldsymbol{u}_{m}$ are the DMD-modes (calculated in each one of the variables analyzed), and $a_{m}, \delta_{m}$, and $\omega_{m}$ represent their associated amplitudes, growth rates, and frequencies, respectively. The dimension $N$ of the vector space (containing the approximation (1.1)) spanned by the DMD modes can be referred to as the spatial complexity, which is such that $N \leqslant \min (J, M)[26]$.

The standard DMD algorithm is briefly described in Section 2.1. This method is based on the Koopman assumption, which relates each snapshot with the subsequent snapshot via a linear operator $\boldsymbol{R}$ as

$\boldsymbol{v}_{k+1} \simeq \boldsymbol{R} \boldsymbol{v}_{k}$ for $k=1, \ldots, K-1$.

The good performance of this method has been put in evidence several times in the literature, either to study flow structures and compute the main frequencies and features contained in complex nonlinear flows $[33-35,24,25]$, or to obtain the linear global modes in the stability analysis of laminar flows $[17,14]$. However, the method does not always give the expected results, especially in transient flows [2] and also in highly noisy complex flows (as it will be presented in this article) or, more generally, when the spatial complexity of the flow is smaller than the spectral complexity $(N<M)$. The spatial complexity is smaller than the spectral complexity, for example, in several periodic and quasi-periodic solutions of the Ginzburg-Landau equation and the Lorenz system [26]. The latter is a third order system of ordinary differential equations (thus, $J=N=3$ ) that, for certain parameter values, exhibits very complex periodic solutions with a large number of harmonics (thus $M \gg 3$ ). Consequently, the spectral complexity $M$ is necessarily larger than the spatial complexity $N$ because $N \leqslant \min (J, M)$.

Le Clainche \& Vega [26] proposed a more general method, called higher order DMD (HODMD), to extend the application of standard DMD to data in which the spatial complexity is smaller than the spectral complexity. In the new method, the assumption (1.2) is substituted by a more general higher order Koopman assumption (or DMD- $d$ assumption), which relates each snapshot with the $d$ previous delayed snapshots via some linear operators $\boldsymbol{R}_{k}$, as

$\boldsymbol{v}_{k+d} \simeq \boldsymbol{R}_{1} \boldsymbol{v}_{k}+\boldsymbol{R}_{2} \boldsymbol{v}_{k+1}+\cdots+\boldsymbol{R}_{d} \boldsymbol{v}_{k+d-1}$ for $k=1, \ldots, K-d$.

For $d=1$, this equation reduces to (1.2) and the DMD- 1 algorithm is equivalent to standard DMD. For $d>1$, instead, the new method is more general. A careful comparison of Eqs. (1.2) and (1.3) shows that though all solutions of (1.2) are of the form (1.1), the converse is not true, namely not all expansions of the form (1.1) satisfy (1.2). The more general Eq. (1.3), instead, is such that its solutions are all of the form (1.1) and, moreover, all expansions of the form (1.1) satisfy (1.3) for appropriate $d$ and appropriate matrices $\boldsymbol{R}_{1}, \ldots, \boldsymbol{R}_{d}$ (see [26], appendix A, theorem D). This is the mathematical essence of the good performance of HODMD.

The HODMD algorithm is briefly summarized in Section 2.2 , as developed in [26]. However, in highly noisy data associated with rather complex flow dynamics, which is more common in experiments, the DMD- $d$ algorithm should be preceeded by a special data cleaning treatment if understanding the flow physics is the main goal of the study. Physical phenomena are generally unknown in many studies [25] and noisy data may lead to calculations of noisy DMD-modes which are difficult to interpret and understand [13] and in some cases, even spurious results can be misinterpreted as flow instabilities.

In this article, the DMD- $d$ algorithm developed in [26] is revisited and modified with the aim at making it suitable for studying flow structures in highly noisy complex data. Noisy data are somewhat cleaned, in both standard DMD and HODMD, in the application of truncated singular value decomposition (SVD), which is always performed as a first step. However, standard SVD is substituted in this paper with higher order SVD (HOSVD). This method considers a tensor encompassed by all field variables (i.e. in a three-dimensional incompressible flow these variables are three in space, $x, y, z$, and one in time $t_{k}$ for each velocity field $u_{x}, u_{y}$ and $u_{z}$ ) and calculates the SVD modes of the associated tensor fibers (higher order analog of the matrix rows and columns). The benefit of this method is the capability to independently clean the data in the different directions (i.e., the three spatial directions and time) of the studied field. Therefore, the combination of HOSVD and HODMD is able to clean not only the temporal modes but also the spatial modes associated with the various spatial directions, producing a less noisy reconstruction of the snapshots than its counterpart obtained upon application of HODMD alone. Moreover, the HOSVD/HODMD combination can be applied iteratively to obtain even cleaner DMD-reconstructions of the noisy snapshots.

This new method is applied to study the flow structures of a zero-net-mass-flux (ZNMF) jet. Time-resolved particle image velocimetry (PIV) experiments were carried out in the near field of the jet, where the flow remains transitional. The high complexity of this flow, in conjunction with the noise produced by the experimental measurements, make this case suitable to test the performance of this new methodology.

The article is organized as follows. Section 2 summarizes the standard DMD and the HODMD algorithms, while the combination of HOSVD and HODMD is fully developed in Section 3. Section 4 is devoted to a description of the ZNMF jet and the experimental set up used to obtain the experimental data. The application of the combined HOSVD/HODMD method to the ZNMF jet flow is considered in Section 5, where a comparison is made with standard DMD. Section 6 contains the analysis of the structures appearing in the ZNMF flow using the HOSVD/HODMD method iteratively, which as anticipated, improves the results. Section 7 identifies the amplitude raise in the spatial growing instabilities that lead to the transition from laminar to turbulent flow in the ZNMF jet. The paper ends with some concluding remarks, in Section 8.

\section{The standard DMD and HODMD algorithms}

The main goal of both standard DMD and HODMD is to compute the expansion (1.1), which is rewritten here as

$$
\boldsymbol{v}_{k} \simeq \boldsymbol{v}_{k}^{\mathrm{DMD}} \equiv \sum_{m=1}^{M} a_{m} \boldsymbol{u}_{m} \mathrm{e}^{\left(\delta_{m}+\mathrm{i} \omega_{m}\right)(k-1) \Delta t} \quad \text { for } k=1, \ldots, K,
$$

Such computation is to be performed using a set of $K$ time equispaced snapshots $\boldsymbol{v}_{k}$ that, for convenience, are collected in the following snapshot matrix

$\boldsymbol{V}_{1}^{K}=\left[\boldsymbol{v}_{1}, \boldsymbol{v}_{2}, \ldots, \boldsymbol{v}_{k}, \boldsymbol{v}_{k+1}, \ldots, \boldsymbol{v}_{K-1}, \boldsymbol{v}_{K}\right]$.

\subsection{Standard DMD}

Beginning with standard DMD, the linear relationship between subsequent snapshots (1.2) can be written in matrix form in two ways, either as

$\boldsymbol{V}_{2}^{K} \simeq \boldsymbol{R} \boldsymbol{V}_{1}^{K-1}$ 
where the Koopman matrix is the finite dimensional counterpart of (1.2) or as

$\boldsymbol{V}_{2}^{K} \simeq \boldsymbol{V}_{1}^{K-1} \mathbf{C}$,

where the companion matrix $\boldsymbol{C}$ is defined as

$\boldsymbol{C} \equiv\left[\begin{array}{ccccc}\mathbf{0} & \mathbf{0} & \ldots & \mathbf{0} & \boldsymbol{c}_{1} \\ -1 & \mathbf{0} & \ldots & \mathbf{0} & \boldsymbol{c}_{2} \\ \mathbf{0} & -1 & \ldots & \mathbf{0} & \boldsymbol{c}_{3} \\ \ldots & \ldots & \ldots & \ldots & \ldots \\ \mathbf{0} & \mathbf{0} & \ldots & -1 & \boldsymbol{c}_{K}\end{array}\right]$.

Schmid [32] describes in detail two different methods to first calculate the matrix $\boldsymbol{R}$ or $\boldsymbol{C}$ appearing in (2.3) and (2.4), and then compute the DMD-modes, growth rates, and frequencies using the eigenvalues and eigenvectors of the matrices $\boldsymbol{R}$ or $\boldsymbol{C}$. In both cases, the pseudoinverse of the snapshot matrix $\boldsymbol{V}_{1}^{K-1}$, is calculated via SVD, but only in the first case it is possible to eliminate spatial redundancies via truncated SVD. On the other hand, only the second method require highly linearly dependent data (i.e., a sufficiently long sequence of snapshots). For convenience, the second method based on the companion method will be called in this article theDMD-companion method.

\subsection{HODMD: the DMD-d algorithm}

As anticipated in Section 1, the new HODMD method is based on the more general assumption (1.3), which can also be written in matrix form as ( $\mathrm{cf}(2.3)$ )

$\boldsymbol{V}_{d+1}^{K} \simeq \boldsymbol{R}_{1} \boldsymbol{V}_{1}^{K-d}+\boldsymbol{R}_{2} \boldsymbol{V}_{2}^{K-(d-1)}+\cdots+\boldsymbol{R}_{d} \boldsymbol{V}_{d}^{K-1}$.

This equation suggests that, as illustrated in Fig. 1, it is possible to compare DMD- $d$ with the sliding window used in the improvement of standard Fourier transform known as power spectral density (PSD) analysis [30], in which the number of involved segments is the counterpart of the parameter $d$ in DMD- $d$. Because of this sliding window process, when the spatial complexity $N$ is smaller than the spectral complexity $M$ (very common in nonlinear dynamical systems), DMD- $d$ enables the calculation of several temporal modes associated with a single spatial mode. This is the fact that leads to the improved performance of DMD- $d$ against standard DMD. Even in cases in which the spatial and spectral complexities coincide $(N=M)$, one can expect to obtain more accurate and less noisy results using DMD- $d$ compared to standard DMD, with a smaller number of snapshots. Alternatively, the time-delayed snapshot matrices appearing in Fig. 1 do have something in common with the use of time-delayed snapshots, which has been repeatedly seen to contribute to increase observability in model identification [4], relaying on the Taken's delay embedding theorem [42].

The computational cost of directly using Eq. (2.6) may be prohibitively expensive if $d$ is large. Thus, to reduce this cost, two dimension-reduction steps are applied previously to the computation of the DMD modes, growth rates, and frequencies. The DMD- $d$ algorithm is implemented in five steps, considered in the following subsections.

\subsubsection{Step 1: First dimension reduction step}

The spatial dimension $J$ of the original data set of snapshots is reduced to a set of linearly independent vectors of dimension $N$. Truncated SVD is applied to the snapshot matrix $\boldsymbol{V}_{1}^{K}$ defined in (2.2) as

$\boldsymbol{V}_{1}^{K} \simeq \boldsymbol{W} \Sigma \boldsymbol{T}^{\top}$,

where $\boldsymbol{W}^{\top} \boldsymbol{W}=\boldsymbol{T}^{\top} \boldsymbol{T}=$ the $N \times N$-unit matrix and the diagonal of matrix $\mathbf{\Sigma}$ contains the singular values $\sigma_{1}, \ldots, \sigma_{K}$. The number of

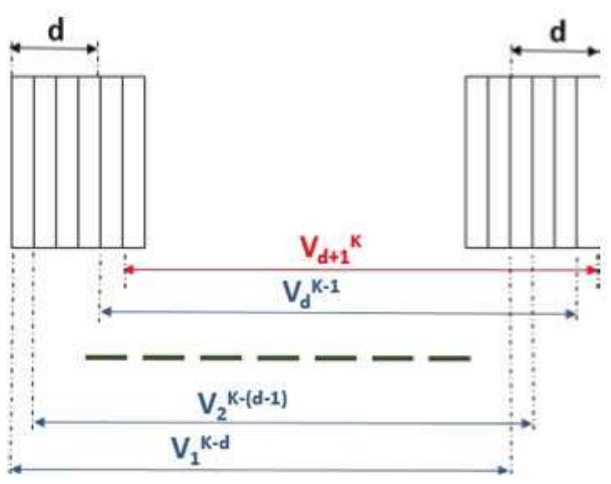

Fig. 1. Snapshot matrix $\boldsymbol{V}_{1}^{K}$ and sliding window process of DMD-d defined in Eq. (1.3). Each column represents a single snapshot $\boldsymbol{v}_{k}$.

retained SVD modes, $N$, is calculated through the standard SVDerror estimate for a certain tolerance $\varepsilon_{t}$ (set by the user), as

$\sigma_{N+1} / \sigma_{1} \leqslant \varepsilon_{t}$.

For convenience, Eq. (2.7) is rewritten as

$\boldsymbol{V}_{1}^{K}=\boldsymbol{W} \hat{\boldsymbol{V}}_{1}^{K}$ with $\hat{\boldsymbol{V}}_{1}^{K}=\mathbf{\Sigma} \boldsymbol{T}^{\top}$.

In other words, the original snapshot matrix $\boldsymbol{V}_{1}^{K}$ is written as product of spatial and temporal terms, $\boldsymbol{W}$ and $\hat{\boldsymbol{V}}_{1}^{K}$, respectively. The latter is called the reduced snapshot matrix, whose size $N \times K$ is generally smaller than that of $\boldsymbol{V}_{1}^{K}$.

\subsubsection{Step 2: Second dimension reduction step}

Now, the higher order Koopman assumption (2.6) is applied to the reduced snapshot matrix. Pre-multiplying (2.6) by $\boldsymbol{W}^{\top}$ yields

$\hat{\boldsymbol{V}}_{d+1}^{K} \simeq \hat{\boldsymbol{R}}_{1} \hat{\boldsymbol{V}}_{1}^{K-d}+\hat{\boldsymbol{R}}_{2} \hat{\boldsymbol{V}}_{2}^{K-d+1}+\cdots+\hat{\boldsymbol{R}}_{d} \hat{\boldsymbol{V}}_{d}^{K-1}$,

where $\hat{\boldsymbol{R}}_{k}=\boldsymbol{W}^{\top} \boldsymbol{R}_{k} \boldsymbol{W}$ for $k=1, \ldots, d$. This equation is rewritten as

$\tilde{\boldsymbol{V}}_{2}^{K-d+1}=\tilde{\boldsymbol{R}} \tilde{\boldsymbol{V}}_{1}^{K-d}$,

where the modified Koopman matrix is the $\mathrm{Nd} \times \mathrm{Nd}$ matrix

$\tilde{\boldsymbol{R}} \equiv\left[\begin{array}{cccccc}\mathbf{0} & \mathbf{I} & \mathbf{0} & \ldots & \mathbf{0} & \mathbf{0} \\ \mathbf{0} & \mathbf{0} & \mathbf{I} & \ldots & \mathbf{0} & \mathbf{0} \\ \ldots & \ldots & \ldots & \ldots & \ldots & \ldots \\ \mathbf{0} & \mathbf{0} & \mathbf{0} & \ldots & \mathbf{I} & \mathbf{0} \\ \hat{\boldsymbol{R}}_{1} & \hat{\boldsymbol{R}}_{2} & \hat{\boldsymbol{R}}_{3} & \ldots & \hat{\boldsymbol{R}}_{d-1} & \hat{\boldsymbol{R}}_{d}\end{array}\right]$

and $\tilde{\mathbf{V}}_{1}^{K-d}$ and $\tilde{\boldsymbol{V}}_{2}^{K-d+1}$ are the sub-matrices formed with the first $K-d$ columns and the last $K-d$ columns of the modified snapshot matrix, defined as

$\tilde{\boldsymbol{V}}_{1}^{K-d+1} \equiv\left[\begin{array}{c}\hat{\mathbf{V}}_{1}^{K-d+1} \\ \hat{\boldsymbol{V}}_{2}^{K-d+2} \\ \cdots \\ \hat{\boldsymbol{V}}_{d-1}^{K-1} \\ \hat{\boldsymbol{V}}_{d}^{K}\end{array}\right]$

This matrix is expected to also exhibit redundancies that are eliminated by a new dimension-reduction via truncated SVD, as

$\tilde{\boldsymbol{V}}_{1}^{K-d+1} \simeq \tilde{\boldsymbol{U}} \tilde{\Sigma} \tilde{\boldsymbol{T}}^{\top} \equiv \tilde{\boldsymbol{U}} \boldsymbol{T}_{1}^{K-d+1} \quad$ with $\boldsymbol{T}_{1}^{K-d+1}=\tilde{\Sigma} \tilde{\boldsymbol{T}}^{\top}$,

where $\tilde{\boldsymbol{U}}^{\top} \tilde{\boldsymbol{U}}=\tilde{\boldsymbol{V}}^{\top} \tilde{\boldsymbol{V}}=$ the $N_{\prime} \times N^{\prime}$-unit matrix and the diagonal of matrix $\hat{\mathbf{\Sigma}}$ contains the singular values $\tilde{\sigma}_{1}, \ldots, \tilde{\sigma}_{N / \text {. }}$ The number of retained SVD modes, $N$, is again calculated as ( $\mathrm{cf}(2.8)$ )

$\tilde{\sigma}_{N^{\prime}+1} / \tilde{\sigma}_{1} \leqslant \varepsilon_{t}$, 
where the tunable threshold $\varepsilon_{\tau}$ coincides with that in (2.8). The step is completed via pre-multiplication of Eq. (2.11) by $\tilde{\boldsymbol{U}}^{\top}$, which invoking (2.14) yields

$\boldsymbol{T}_{2}^{K-d+1}=\overline{\boldsymbol{R}} \boldsymbol{T}_{1}^{\boldsymbol{K}-\boldsymbol{d}}$,

where the new modified $\mathrm{N} I \times \mathrm{N} /$-Koopman matrix is defined as $\overline{\boldsymbol{R}}=\hat{\boldsymbol{U}}^{\top} \tilde{\boldsymbol{R}} \tilde{\boldsymbol{U}}$. However, this latter equation will not be used below. Instead, $\overline{\boldsymbol{R}}$ is computed in the next step from Eq. (2.16).

\subsubsection{Step 3: Computation of the reduced DMD modes, growth rates,} and frequencies

As in standard DMD, the matrix $\overline{\boldsymbol{R}}$ is obtained by using the pseudo-inverse of matrix $\boldsymbol{T}_{1}^{K-d}$, which is done via plain (no truncation) SVD as

$\boldsymbol{T}_{1}^{K-d}=\boldsymbol{U} \boldsymbol{\Lambda} \boldsymbol{V}^{\top}$

where the diagonal of $\boldsymbol{\Lambda}$ contains the singular values and $\boldsymbol{U U}^{\top}=\boldsymbol{U}^{\top} \boldsymbol{U}=\boldsymbol{V}^{\top} \boldsymbol{V}=$ the $N^{\prime} \times N^{\prime}$-unit matrix. Substituting (2.17) into (2.16) and post-multiplying the resulting equation by $\boldsymbol{V}_{\boldsymbol{A}}^{-1} \boldsymbol{U}^{\top}$ yields

$\bar{R}=T_{2}^{K-d+1} V \Lambda^{-1} U^{\top}$.

Once the matrix $\overline{\boldsymbol{R}}$ has been calculated, we note that (2.16) is the similar to the Eq. (2.3) involved in standard SVD. Namely, the columns of the snapshot matrices appearing in (2.16) approximately satisfy $\boldsymbol{t}_{k+1}=\overline{\boldsymbol{R}} \boldsymbol{t}_{k}$, which means that they are such that

$\boldsymbol{t}_{k} \simeq \sum_{m=1}^{M} a_{m} \boldsymbol{q}_{m} \mathrm{e}^{\left(\delta_{m}+\mathrm{i} \omega_{m}\right)(k-1) \Delta t}$ for $k=1, \ldots, K-d+1$,

where $\boldsymbol{q}_{m}$ are the eigenvectors of $\overline{\boldsymbol{R}}$. The associated eigenvalues, $\mu_{m}$, yield the growth rates $\delta_{m}$ and frequencies $\omega_{m}$ appearing in this expansion as

$\delta_{m}+\mathrm{i} \omega_{m}=\log \left(\mu_{m}\right) / \Delta t$

Collecting the second dimension-reduction performed in Section 2.2.2, namely invoking (2.13) and (2.14), the expansion (2.19) readily leads to its counterpart from the reduced snapshots (i.e., the columns of the reduced snapshot matrix $\hat{\boldsymbol{V}}_{1}^{K-d+1}$ ),

$\hat{\boldsymbol{v}}_{k} \simeq \sum_{m=1}^{M} a_{m} \hat{\boldsymbol{u}}_{m} \mathrm{e}^{\left(\delta_{m t}+\mathrm{i} \omega_{m}\right)(k-1) \Delta t}$ for $k=1, \ldots, K-d+1$,

where $\delta_{m}$ and $\omega_{m}$ coincide with their counterparts in (2.19). The reduced DMD modes $\hat{\boldsymbol{u}}_{m}$ are the vectors obtained from their counterparts appearing in (2.19) by retaining only the first $\mathrm{N}$ components of the vectors $\tilde{\boldsymbol{q}}_{m}=\tilde{\boldsymbol{U}} \boldsymbol{q}_{m}$, whose size is $\mathrm{Nd}$. The mode amplitudes $a_{m}$ remain undetermined in this step.

\subsubsection{Step 4: Computation of the mode amplitudes}

The mode amplitudes of the DMD expansion (2.21) are calculated vis least-squares fitting (as in optimized DMD method [8]) applied to the $K$ equations that result from extending (2.21) to the indexes $k=1, \ldots, K$. This least-squares problem leads to

$\boldsymbol{L} \boldsymbol{a}=\boldsymbol{b}$

Here, the $N K \times N^{\prime}$-matrix $\boldsymbol{L}$, the unknown amplitudes vector $\boldsymbol{a}$, and the forcing term $\boldsymbol{b}$ are given by

$\boldsymbol{L}=\left[\begin{array}{c}\mathbf{Q} \\ \boldsymbol{Q} \boldsymbol{M} \\ \ldots \\ \boldsymbol{Q} \boldsymbol{M}^{K-1}\end{array}\right], \quad \boldsymbol{a}=\left[\begin{array}{c}a_{1} \\ a_{2} \\ \ldots \\ a_{M}\end{array}\right], \quad \boldsymbol{b}=\left[\begin{array}{c}\hat{\boldsymbol{v}}_{1} \\ \hat{\boldsymbol{v}}_{2} \\ \ldots \\ \hat{\boldsymbol{v}}_{K}\end{array}\right]$. where $\boldsymbol{Q}=\left[\hat{\boldsymbol{u}}_{1}, \ldots, \hat{\boldsymbol{u}}_{M}\right]$ and the diagonal matrix $\boldsymbol{M}$, of dimension $\mathrm{N} / \times N /$, contains in its diagonal the exponentials $\mathrm{e}^{\left(\delta_{1}+i \omega_{1}\right) \Delta t}, \ldots, \mathrm{e}^{\left(\hat{\delta}_{M}+\mathrm{i} \omega_{M}\right) \Delta t}$. To calculate the amplitude vector $\boldsymbol{a}$ it is necessary to apply the pseudo-inverse of matrix $\boldsymbol{L}$, which is done via plain SVD (no truncation).

Finally, the DMD modes are reordered by sorting the mode amplitudes in decreasing order, and the retained number of modes, $M \leqslant N t$, is selected as the largest value of $M$ such that

$a_{M+1} / a_{1}<\varepsilon_{a}$,

for some threshold $\varepsilon_{a}$ set by the user.

\subsubsection{Step 5: Computation of the DMD expansion for the original} snapshots: calculation of the DMD modes

Invoking Eq. (2.9), the DMD expansion (2.1) readily follows from (2.21), with the DMD modes defined as

$\boldsymbol{u}_{m}=\boldsymbol{W} \hat{\boldsymbol{u}}_{m}$

while the mode amplitudes, growth rates, and frequencies coincide with their counterparts in (2.21). This completes the step and the summary of the method.

\section{Combination of HOSVD and HODMD}

In the above, the various snapshots in the DMD expansion (1.1) have been considered as vectors that, for fluid flows, collect all velocity components in the various spatial mesh points. In fact, the DMD expansion (1.1) can be written componentwise as

$V_{j k} \simeq \sum_{m=1}^{M} a_{m} U_{j m} \mathrm{e}^{\left(\delta_{m}+\mathrm{i} \omega_{m}\right)(k-1) \Delta t}$ for $j=1, \ldots, J, k=1, \ldots, K$,

However, when the spatial mesh is structured, the velocity components may be considered separately and dependence on the various spatial coordinates may be considered separately too. Then, the snapshots conform to a multidimensional array, depending on more than two indexes, namely a tensor (not a matrix), which will be called below the snapshot tensor. The counterparts of the rows and columns of the matrix are known as the fibers of the tensor. For instance, for a third order tensor, the fibers are as illustrated in Fig. 2.

The snapshot tensor depends on the flow topology and the computational mesh, and can be organized in various ways. For instance, for the time-dependent ZNMF jet considered below, PIV measurements in a plane containing the symmetry axis give the radial and streamwise velocity components, $v_{\mathrm{r}}$ and $v_{x}$, of the inplane velocity $v$ in a $J_{2} \times J_{3}$-Cartesian coordinate system, as

$\boldsymbol{v}\left(r_{j_{2}}, x_{j_{3}}, t_{k}\right)$ for $j_{2}=1, \ldots, J_{2}, j_{3}=1, \ldots, J_{3}, k=1 \ldots, K$

Thus, the snapshots can be organized in a fourth-order $2 \times J_{2} \times J_{3} \times K$-tensor $\boldsymbol{V}$, whose components $V_{j_{1} j_{2} j_{3} k}$ are defined as

$V_{1 j_{2} j_{3} k}=v_{r}\left(r_{j_{2}}, x_{j_{3}}, t_{k}\right), \quad V_{2 j_{2} j_{3} k}=v_{x}\left(r_{j_{2}}, x_{j_{3}}, t_{k}\right)$.

In other words, the index $j_{1}$ labels the two velocity components, the indexes $j_{2}$ and $j_{3}$ label the discrete values of the two spatial coordinates, and $k$ labels the considered equispaced values of time. In this context, the DMD expansion (3.1) must be substituted by its tensor counterpart

$V_{j_{1} j_{2 j} j_{3}} \simeq \sum_{m=1}^{M} a_{m} U_{j_{1} j_{2} j_{3} m} \mathrm{e}^{\left(\delta_{m}+\mathrm{i} \omega_{m}\right)(k-1) \Delta t}$

Note that this expansion leads to (3.1) when the first three indexes, $j_{1}, j_{2}$, and $j_{3}$, are folded together into a single index $j$. However, as anticipated, the tensor form (3.4) permits substituting SVD by HOSVD (described in the next subsection) in the dimension reduc- 

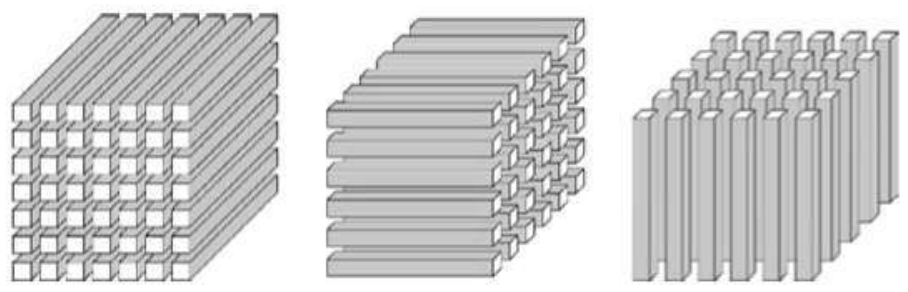

Fig. 2. The fibers of a third order tensor.

tion step of the HODMD calculations, which leads to a more efficient noise filtering method.

For the sake of clarity, the remainder of this section is concerned with the fourth order tensor defined in (3.3), though the extension to higher order tensors is straight forward. For instance, fully three-dimensional descriptions of time-dependent flows, discretized in a $J_{2} \times J_{3} \times J_{4}$ structured spatial mesh (resulting from, e.g., numerical simulations) lead to fifth-order tensors of size $3 \times J_{2} \times J_{3} \times J_{4} \times K$.

\subsection{Higher order singular value decomposition}

The HOSVD algorithm was introduced by Tucker [43] in 1966 and has been more recently popularized by de Lathauwer et al. $[9,10]$. This algorithm has been successfully used in a variety of fields, including generation [27] and compression [28] of aeronautic databases, real-time control of automotive engines [3], and conceptual aeronautic design [11]. The HOSVD of the fourth order tensor defined in (3.3) is given by

$V_{j_{1} j_{2} j_{3} k} \simeq \sum_{p_{1}=1}^{P_{1}} \sum_{p_{2}=1}^{P_{2}} \sum_{p_{3}=1}^{P_{3}} \sum_{n=1}^{N} S_{p_{1} p_{2} p_{3} n} W_{j_{1} p_{1}}^{(1)} W_{j_{2} p_{2}}^{(2)} W_{j_{3} p_{3}}^{(3)} T_{k n}$

where $\boldsymbol{S}_{p_{1} p_{2} p_{3} n}$ is another fourth-order tensor (called the core tensor) and the columns of the matrices $\boldsymbol{W}^{(1)}, \boldsymbol{W}^{(2)}, \boldsymbol{W}^{(3)}$, and $\boldsymbol{T}$ are known as the modes of the decomposition. Note that the first three sets of modes (i.e., the columns of the matrices $\boldsymbol{W}^{(t)}$ for $l=1,2$, and 3 ) account for dependence on the spatial variables, while the fourth set of modes (i.e., the columns of the matrix $\boldsymbol{T}$ ) is connected with the time variable. The four sets of modes are obtained by applying standard SVD to the four matrices whose columns are the associated fibers of the tensor. The associated four sets of non-zero singular values of the decomposition are sorted in decreasing order and denoted as

$\sigma_{p_{1}}^{(1)}, \quad \sigma_{p_{2}}^{(2)}, \quad \sigma_{p_{3}}^{(3)}, \quad$ and $\sigma_{n}^{t}$.

Once the modes have been calculated, the core tensor is given by

$S_{p_{1} p_{2} p_{3} n}=\sum_{j_{1}=1 j_{2}=1}^{J_{1}} \sum_{j_{3}=1}^{J_{2}} \sum_{k=1}^{J_{3}} \sum_{j_{1} j_{2} j_{3} k}^{K} \hat{W}_{j_{1} p_{1}}^{(1)} \hat{W}_{j_{2} p_{2}}^{(2)} \hat{W}_{j_{3} p_{3}}^{(3)} \hat{V}_{k n}$,

as readily obtained by multiplying (3.5) by $\hat{W}_{j_{1} p_{1}}^{(1)} \hat{W}_{j_{2} p_{2}}^{(2)} \hat{W}_{j_{3} p_{3}}^{(3)} \hat{V}_{k n}$, adding the resulting equation in $j_{1}, j_{2}, j_{3}$, and $k$, and taking into account that the modes are orthonormal. The computation of the core tensor, singular values, and modes are very efficiently performed using the MATLAB function 'hosvd' ${ }^{1}$ (provided in the file exchange of MATLAB community as toolbox TP Tool), which is able to treat tensors of any order. Note that without truncation the HOSVD (3.5) is exact. Upon truncation, the decomposition is only approximate, but it benefits from the noise filtering effect resulting from the four SVD applications.

\footnotetext{
${ }^{1}$ https://es.mathworks.com/matlabcentral/fileexchange/25514-tp-tool/content/tp-
} tool/array/hosvd.m
Once truncation has been applied, the HOSVD (3.1) is rewritten as

$V_{j_{1} j_{2} j_{3} k} \simeq \sum_{n=1}^{N} W_{j_{1} j_{2} j_{3} n} \hat{V}_{k n}$

where $N$ is the spatial complexity defined above, which determines the (common) number of spatial modes $W_{j_{1} j_{2} j_{3} n}$ and rescaled temporal modes $\hat{V}_{k n}$, defined as

$W_{j_{1} j_{2} j_{3} n}=\sum_{p_{1}=1}^{P_{1}} \sum_{p_{2}=1}^{P_{2}} \sum_{p_{3}=1}^{P_{3}} S_{p_{1} p_{2} p_{3} n} W_{j_{1} p_{1}}^{(1)} W_{j_{2} p_{2}}^{(2)} W_{j_{3} p_{3}}^{(3)} / \sigma_{n}^{t}, \quad \hat{V}_{k n}=\sigma_{r}^{t} T_{k n}$.

Note that the temporal modes (namely, the columns of the matrix $\boldsymbol{T}$ ) have been rescaled with the associated temporal singular values, as we did in (2.9) when the DMD- $d$ algorithm was applied to twodimensional data. As in Section 2.2, we apply the steps 2-4 of the DMD- $d$ algorithm to the reduced snapshot matrix $\hat{\boldsymbol{V}}$, which yields a reduced DMD expansion, which for convenience is written here in matrix form as

$\hat{V}_{k n} \simeq \sum_{m=1}^{M} a_{m} \hat{U}_{m n} \mathrm{e}^{\left(\delta_{m}+\mathrm{i} \omega_{m}\right)(k-1) \Delta t}$ for $k=1, \ldots, K$.

Substituting this into (3.8) readily yields the multidimensional DMD expansion (3.4), with

$U_{j_{1} j_{2} j_{3} m}=\sum_{n=1}^{N} W_{j_{1} j_{2} j_{3} n} \hat{U}_{m n}$

This completes the description of the method, which is summarized in the next subsection.

\subsection{Summary of the multidimensional DMD-d algorithm}

This algorithm is very efficiently implemented in MATLAB, using the following functions provided in the file exchange of MATLAB community as toolbox TP Tool: 'hosvd' (to calculate the core tensor and the modes appearing in the HOSVD expansion (3.5)), 'tprod' (to perform the products appearing in (3.7)-(3.9) and (3.11)), and 'ndim_fold'and 'ndim_unfold' (to fold and unfold indexes, when needed). The algorithm proceeds in two steps:

- Step 1: Application of truncated HOSVD.

1.1 Organize the snapshots as the $2 \times J_{1} \times J_{2} \times J_{3} \times K$ snapshot tensor $\boldsymbol{V}$, with components denoted as $V_{j_{1} j_{2} j_{3} k}$, defined in (3.3).

1.2 Apply plain HOSVD (no truncation) to the snapshot tensor. Note that, for the tensor $\boldsymbol{V}$, the numbers of modes along the various tensor directions are the ranks of the matrices whose columns are the fibers, which at most are $P_{1}=J_{1}=2, P_{2}=\min \left\{J_{2}, 2 J_{3} K\right\}, P_{3}=\min \left\{J_{3}, 2 J_{2} K\right\}$, and $N=\min \left\{K, 2 J_{2} J_{3}\right\}$. The resulting sets of singular values are denoted as in (3.6). 
1.3 Set the spatial and temporal tolerances $\varepsilon_{s}$ and $\varepsilon_{t}$ to select the numbers of retained modes along the various directions, $P_{1}, P_{2}, P_{3}$, and $N$, as the smallest values such that

$$
\sigma_{P_{i}+1}^{(l)} / \sigma_{1}^{(l)}>\varepsilon_{s} \text { for } l=1,2,3, \quad \sigma_{N+1}^{t} / \sigma_{1}^{t}>\varepsilon_{t} .
$$

1.4 Apply truncated HOSVD retaining the numbers of modes determined in the last item. This gives the core tensor and the modes that define the truncated HOSVD in the right hand side of (3.5).

1.5 Calculate the spatial and temporal modes defined in Eq. (3.9).

- Step 2: Calculation of the multidimensional DMD expansion.

2.1 The steps 2-4 in the DMD- $d$ algorithm described in Section 2.2 are applied to the reduced snapshot matrix defined by the temporal modes $\hat{V}_{k r}$ calculated in the previous step, item 1.5, which gives the mode amplitudes $a_{m}$. reduced modes $\hat{U}_{m n}$, growth rates $\delta_{m}$, and frequencies $\omega_{m}$ appearing in (3.10). Note that, in particular, the amplitudes $a_{m}$ are calculated by least square fitting of the two sides of (3.10).

2.2 Use Eq. (3.11) to compute the DMD modes appearing in the multidimensional DMD expansion (3.4). The amplitudes, growth rates, and frequencies are as calculated in the previous item.

2.3 Rescale the mode amplitudes and modes such that the root-mean-square (RMS) norm of the modes is one namely

$$
\sqrt{\frac{1}{J_{1} J_{2} J_{3}}}\left|U_{j_{1} j_{2} j_{3} m}\right|=1 \text { for } m=1, \ldots, M \text {. }
$$

Reorder the modes for decreasing values of $\left|a_{m}\right|$. Then the DMD expansion (3.4) is truncated by neglecting those terms such that

$$
a_{m} / a_{1} \leqslant \varepsilon_{a}
$$

for some tunable tolerance $\varepsilon_{a}$.

This is the basic algorithm that will be applied to the ZNMF jet in Section 5, where the method will be compared with standard DMD.

\subsection{Comments and iterative extension}

The muti-dimensional DMD algorithm depends on four tunable parameters:

- The index $d$ that defines the DMD- $d$ algorithm applied in step 2.1. As further explained in [26], a good way to determine the optimal value of $d$ is to prove the robustness of the results. For example, when $d$ is optimal, the same results must be obtained when the method is applied in two different time intervals. Usually, there is a wide range of values of $d$ (which varies with the tolerances $\varepsilon_{t}$ and $\varepsilon_{a}$, the number of data collected, and their distance $\Delta t$ ) that provides the same good results. Depending on the application, the optimal value of $d$ lies somewhere in between of $d=1$ (when the spatial complexity $N$ equals the spectral complexity $M$ ) and a value $d \sim K / 3$ (when $M$ is larger than $N$ ). Note that $d$ should be not too large because the first $d$ snapshots are only used in (2.6) as initial conditions and are thus lost in the DMD computations. Also, for a given sampled time interval, the optimal value of $d$ scales with the total number of snapshots, $K$, namely $d$ should be doubled if $K$ is multiplied by 2 .
- The spatial and temporal tolerances $\varepsilon_{s}$ and $\varepsilon_{t}$ govern truncation on space and time, see Eq. (3.12), and the tolerance $\varepsilon_{a}$ governs truncation on the mode amplitudes, defined in Eq. (3.14). When treating experimental data, $\varepsilon_{s}, \varepsilon_{t}$, and $\varepsilon_{a}$ should be comparable to the expected uncertainty of the measurements.

It is convenient to note that if no spatial truncation is made (namely, if truncation is performed only in the temporal index $k$ ), then the multidimensional DMD expansion (3.4) exactly coincides with its two-dimensional counterpart (3.1) after folding the first three indexes in (3.4), into a single index $j$. Thus, the role of the spatial truncation (in the indexes $j_{1}, j_{2}$, and $j_{3}$ ) is to filter out noise in the spatial directions. After this filtering, the method can be iterated. As a first step, the multidimensional DMD method itself is applied again, using the same tolerances as in the first application, to the (cleaner) reconstructed snapshots (according to the right hand side of (3.4)) that are obtained in the first application of the multidimensional DMD method. As a second step, the same algorithm is applied to the newly reconstructed snapshots, and so on. The iterative process stops when the number of HOSVD modes are maintained after two consecutive iterations. The main advantage of the iterative methodology lies in the omission of irrelevant or inconsistent HOSVD modes according to an established tolerance. Each time the algorithm is applied to the reconstructed snapshot tensor, both HOSVD and DMD- $d$ modes are recalculated and ordered as a function of their new corresponding amplitudes. Thus, the iterative method subsequently improves the quality of the DMD reconstructions; see Section 6.

Such iteration could also be applied using the two-dimensional DMD expansion (3.1), but the efficiency of the resulting method would be limited.

\section{Application to the analysis of a zero-net-mass-flux jet}

A zero-net-mass-flux (ZNMF) jet is a fluid stream that is formed by the interaction of vortex rings that separate from the exit of a jet orifice. ZNMF jets are generated by the periodic oscillations of a membrane or a piston in a cavity. These oscillations cause the flow to leave the cavity through a small orifice and go back into the cavity through the same orifice periodically. Thus, the net mass flow through the orifice is zero. When the flow passes through the jet orifice in the injection phase, the sharp edges of this orifice result in the flow separating and rolling up to form a vortex ring that propagates downstream due to its self-induced velocity. In the suction phase the flow is sucked through the orifice. However, the vortex ring produced in the former injection phase is not affected by suction since the vortex is sufficiently far away to continue its downstream movement [41].

The vortices are formed from the working fluid of the flow system in which they develop without the net injection of additional fluid, which makes this kind of jets suitable for several applications such as to enhance mixing and heat transfer or to control flow separation [16,7]. Therefore, the flow generation mechanism (piston or membrane) produces a non-zero mean streamwise momentum formed by the interaction of the vortices that travel downstream and transfer energy to the environment. This fact suggests that the velocity scale of a ZNMF jet should be based on mean momentum [5].

The vortex ring produced in the ZNMF jet is initially laminar, but it transitions to turbulent flow. Smith and Glezer [36] suggest that, in a ZNMF jet with a square cavity, this transition is due to wavy azimuthal instability that causes vortex breakdown, similarly to the inviscid spanwise instability observed by Widnall and Tsai [45]. 


\subsection{Geometry and flow parametrization}

The present investigation studies the flow structures in the near field of a round ZNMF jet. This jet is generated by a piston oscillating in a cylindrical cavity behind a circular orifice. Fig. 3 shows a two-dimensional sketch of the geometry. The jet is referred to a cylindrical coordinate system, whose radial, streamwise, and azymuthal coordinates are denoted as $r, x$, and $\theta$, respectively, and the associated velocity components as $v_{r}, v_{x}$, and $v_{\theta}$, respectively. The dimensions of the piston and jet orifice diameters are $D_{p}=50 \mathrm{~mm}$ and $D_{o}=10 \mathrm{~mm}$, respectively, the thickness of the jet orifice is $e=2 \mathrm{~mm}$. The piston stroke is a maximum at the distance $L_{c}=3 D_{p}$. The diameter of the jet orifice is considered as the characteristic length scale, while the characteristic velocity scale $U_{0}$ is based on the mean momentum velocity [5], defined as

$U_{0}=\left[\frac{1}{A_{0} T} \int_{0}^{T} \int_{0}^{D_{0} / 2} \int_{0}^{2 \pi} 2 \pi r v(r, \theta, t) d \theta d r d t\right]^{1 / 2}$

in which $A_{o}=\pi D_{o}^{2} / 4$ is the area of the jet orifice, $T$ is the piston oscillation period, and $v(r, \theta, t)$ is the streamwise velocity at the exit of the orifice, $x=0$. In principle, computing $v$ would require integrating the governing equations, which are the incompressible Navier-Stokes equations. However, the momentum velocity (4.1) is defined in this paper using the velocity of the piston $V_{p}(t)$, which is harmonic and given by $V_{p}(t)=\hat{V}_{p} \sin \left(\frac{2 \pi t}{T}\right)$, where $\hat{V}_{p}$ is the amplitude. Thus, the momentum velocity is calculated in terms of the peak piston velocity $\hat{V}_{p}$ as

$U_{o}=\frac{D_{p}}{D_{0}} \frac{\hat{V}_{p}}{\sqrt{2}}$.

The ZNMF jet is characterized by the flow length, velocity, and time scales. Therefore, two non-dimensional parameters are defined for the present investigation: the Reynolds number, defined as $\operatorname{Re}=\frac{U_{0} D_{Q}}{v}$ ( $v$ is the kinematic flow viscosity), and the Strouhal number, defined as $S t=\frac{f D_{\Omega}}{U_{a}}$, where $f=\frac{1}{T}$ is the frequency of the piston oscillations [41]. Based on the diagram presented by Carter and Soria [5] that classifies as function of Re and St the laminar and turbulent description of the flow on a ZNMF jet in the near jet region, this jet has been studied at $R e=13329$ and $S t=0.03$ (with a piston oscillation frequency of $f=4 \mathrm{~Hz}$ ), which lies in the region between laminar and transitional jets in the near field. Consequently the jet is expected to be laminar in the near field, but promoting transition to turbulence in the far field.

The parameter $\phi$ defines the position of the piston in an oscillation cycle, with the origin $\phi=0$ at the position of maximum stroke $\left(L_{c}=3 D_{p}\right)$. The injection and suction phases correspond to the intervals $0<\phi<\pi$ and $\pi<\phi<2 \pi$, respectively.

\subsection{Experimental facility}

Time-resolved 2C-2D PIV experiments have been carried out in the LTRAC ZNMF jets experimental facility at Monash University, previously described in $[18,41]$. Fig. 4 shows the experimental setup of the water tunnel where the experiments were carried out. A ZNMF jet is generated by a piston oscillating in a cavity behind a circular orifice that is connected to an AC motor, which provides the motion of the piston through a flexible coupling and an eccentric plate. Desirable values of $R e$ and St can be obtained by increasing or decreasing the eccentric radius of the plate and the driven frequency $f$. The plane of measurements is parallel to the axis of the jet.

The PIV measurements were performed using a PCO-DIMAX high speed $C C D$ camera, operated in double shutter mode with the maximum acquisition rate of 624 full-frame image pairs per

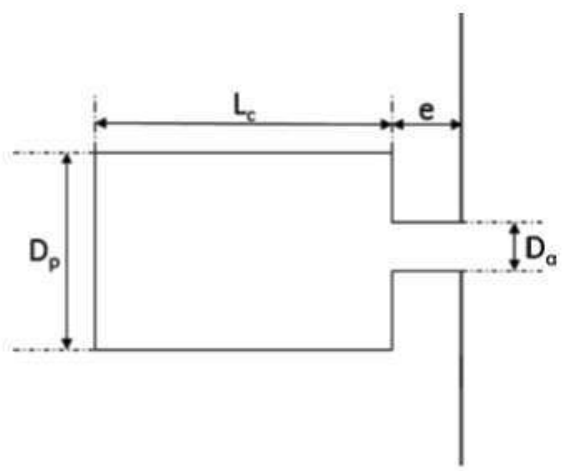

Fig. 3. Sketch of the geometry and parameters of a ZNMF jet.

second. The laser pulse current and the time difference between the two laser pulses was set to $32 \mathrm{~A}$ and $20 \mu \mathrm{s}$, respectively. The axial field of view investigated the near field of the ZNMF jet which extended up to a distance of $3.05 D_{o}$ from the orifice plate. The experimental parameters are listed in Table 1.

Five sets of 3121 image pairs each were acquired, requiring a total of $0.25 \mathrm{~TB}$ of data storage. Each set corresponds to 20 periods of the piston oscillations, and each period is represented by 156 velocity fields. Multigrid cross-correlation particle image velocimetry (MCCDPIV) $[39,40]$ was used to extract the in-plane velocity vector field and out-of-plane vorticity field from this image data. The cross-correlation PIV analysis parameters are summarized in Table 2 . The uncertainty relative to the maximum velocity in the velocity components at the $95 \%$ confidence level is $0.3 \%$ [39]. A local least-squares fit (thirteen point twodimensional fit) procedure was applied to the velocity field to filter the data obtained in the experiment $[39,15]$. This calculation is an approximation that introduces a bias and random error in the vorticity. In the present investigation, it was found that this error is comparable to the random error calculated in the outof-plane vorticity field when it is computed from the fitted velocity using analytic differentiation. This random error is estimated as $+2.4 \%$ at the $95 \%$ confidence level for a vorticity distribution of $0.191 D_{0}$. Further details of this experiment can be found in [41].

\section{Comparison of DMD methods in the ZNMF jet}

The above mentioned experimental data sets are organized in snapshot tensors $\boldsymbol{V}$, as defined in (3.3), where the indexes $j_{2}, j_{3}$, and $k$ correspond to the radial, streamwise, and temporal directions, $r, x$, and $t$, respectively, and will be dimension-reduced via HOSVD in various ways, as explained below. The index $j_{1}$ labels the two velocity components with $j_{1}=1$ and 2 , corresponding to $v_{r}$ and $v_{x}$, respectively, and will not admit any reduction.

For convenience, invoking the definition (3.3) of the snapshot tensor $\boldsymbol{V}$, the DMD expansion (3.4) can be written as

$\boldsymbol{v}\left(r_{j_{2}}, x_{j_{3}}, t_{k}\right) \simeq \sum_{m=1}^{M} a_{m} \boldsymbol{u}^{m}\left(r_{j_{2}}, x_{j_{3}}\right) \mathrm{e}^{\left(\delta_{m}+\mathrm{i} \wp_{m}\right) t_{k}} \quad$ with $t_{k}=(k-1) \Delta t$,

where the radial and streamwise components of the vectors associated with the snapshots and DMD modes, $\boldsymbol{v}=\left(v_{r}, v_{x}\right)$ and $\boldsymbol{u}^{m}=\left(\boldsymbol{u}_{\mathrm{r}}^{m}, u_{x}^{m}\right)$, respectively, are calculated in terms of the tensors appearing in (3.4) as

$v_{r}\left(r_{j_{2}}, x_{j_{3}}, t_{k}\right)=V_{1 j_{2} j_{3} k}, \quad v_{x}\left(r_{j_{2}}, x_{j_{3}}, t_{k}\right)=V_{2 j_{2} j_{3} k}$,

$u_{r}^{m}\left(r_{j_{2}}, x_{j_{3}}\right)=U_{1 j_{2} j_{3} m}, \quad u_{x}^{m}\left(r_{j_{2}}, x_{j_{3}}\right)=U_{2 j_{2} j_{3} m}$. 


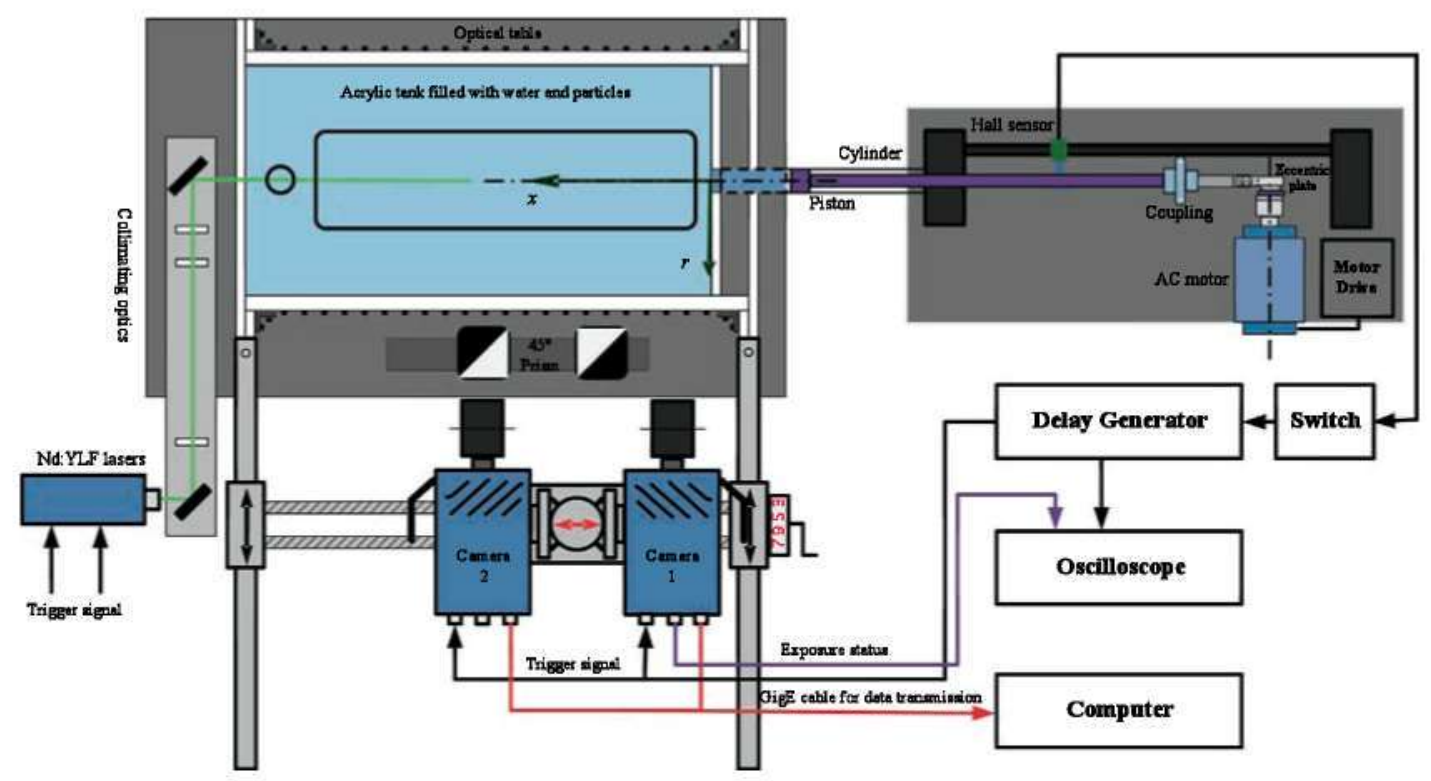

Fig. 4. Experimental setup. Top view of a sketch of the LTRAC ZNMF jet facility. Image extracted from [41].

Table 1

Parameters of the PIV experimental setup,

\begin{tabular}{lc}
\hline Camera & $2016 \times 2016$ pixel, 16 bit \\
Laser & Nd: YLF $527 \mathrm{~nm}$ \\
Laser sheet thickness & $1 \mathrm{~mm}$ \\
Separation time $(\Delta T)$ & $1.6 \mathrm{~ms}$ \\
Flow seeding & $11 \mu \mathrm{m}$ hollow glass spheres, $\rho=1100 \mathrm{Kg} / \mathrm{m}^{3}$ \\
\hline
\end{tabular}

The object of this section is to compare the performance of the standard DMD methods mentioned in Section 1 with the multidimensional DMD- $d$ algorithm summarized in Section 3.2. Thus comparison will be made in terms of the DMD amplitudes/frequencies (in Section 5.1) and reconstructions (in Section 5.2). The snapshot tensor is defined in this section with $K=1872$ snapshots (equivalent to 12 complete cycles of the piston oscillations).

\subsection{DMD amplitudes and frequencies}

After some calibration (following the comments in Section 3.3), the index $d=700(\sim K / 3)$ and the tolerances $\varepsilon_{S}=\varepsilon_{t}=\varepsilon_{a}=0.024$ (corresponding to the uncertainty $\pm 2.4 \%$ of the measurements) have been chosen for the application of the multidimensional DMD method in a set of 1872 snapshots (representing only 12 piston cycles from a total of 20 cycles per data set). In the calibration process it was checked that the same results were obtained in different time intervals for the specific tolerance and $\Delta t$. With this in mind, since DMD-d is an algorithm based on robustness, the minimum suitable number of snapshots and $d$ were selected to perform the analysis.

Fig. 5 gives the mode amplitudes for the corresponding frequencies of the DMD modes retained when using DMD-1, DMD-700, and DMD-companion. Note that, as expected, the HOSVD modes retained in DMD-1 and DMD-700 are the same, since the spatial tolerance is maintained. These are $P_{2}=19, P_{3}=315$, and $N=19$ in $r, x$ and $t$, respectively. However, the number $M$ of DMD modes in these two cases are quite different, namely $M=11$ in DMD-1 and $M=33$ DMD-700. DMD-1 produces completely spurious reconstructions, omitted in the next subsection for the sake of brevity. In contrast, DMD-700 captures the steady mode associated with the mean flow $(S t=0)$, the first harmonic oscillating with the piston oscillation frequency $S t=0.03$, plus its complex conjugate mode, and 13 harmonics of the fundamental frequency (again, plus their complex conjugates). Fig. 5 also shows that the mode amplitudes exhibit spectral decay. There are two additional pairs of complex conjugate modes that could be considered as low accuracy or spurious, one with $S t=0.27$ and another with $S t=0.51$, which duplicate already existing modes with higher amplitudes. Instead, however, the 15th and 16th harmonics are missing in the calculations. These are the only spurious artifacts produced by the method. On the other hand, the accuracy of these results is very high in connection with the frequency, since the relative error that compares the exact frequency of piston oscillations $\left(f_{\text {exact }} \sim S t=0.03\right)$ and the calculated fundamental frequency $\left(f_{D M D}\right)$, measured as $E_{f}=\frac{f_{\text {exact }}-f_{\text {Das }}}{f_{\text {erexp }}} \cdot 100$, is $0.1 \%$. Moreover, the ratio between the frequencies of the highest and lowest amplitude harmonics ( $1 \mathrm{st}$ and $14 \mathrm{th}$ ) computed by DMD, $R_{f D M D}$, is very close to its exact counterpart, $R_{\text {fexact }}=f_{14} / f_{1}=14$. Namely, the relative error of this computation, defined as $E_{R}=\frac{\left|R_{\text {froxat }}-R_{\text {DिMo }}\right|}{R_{\text {frexct }}} \cdot 100$, is $0.01 \%$. These harmonics are related to the highest and lowest amplitude modes, this error can be considered as maximum in connection with the accuracy of the calculations, since this accuracy decreases with the amplitude level (let us remember that tolerances $\varepsilon_{t}, \varepsilon_{s}$, and $\varepsilon_{t}$ are set according to the data uncertainty of the experiments).

Fig. 5 also shows the outcomes of the DMD-companion method, which retains 87 modes that can be classified into two kinds. On the one hand, 25 of these modes show spectral decay (except for two harmonics that are duplicated and one harmonic that is missing, as it happened with the DMD-700 method) and correspond to

Table 2

PIV Cross-correlation parameters (IW: Interrogation Window).

\begin{tabular}{|c|c|c|c|c|}
\hline Parameter & $\Delta T(\mu \mathrm{s})$ & Grid spacing (px) & $I W_{0}(\mathrm{px})$ & $W_{1}(\mathrm{px})$ \\
\hline Quantity & 20 & $16\left(0, \ldots, D_{0}\right)$ & $32(\mathrm{C} 2)$ & $24(\mathrm{C} 2)$ \\
\hline
\end{tabular}




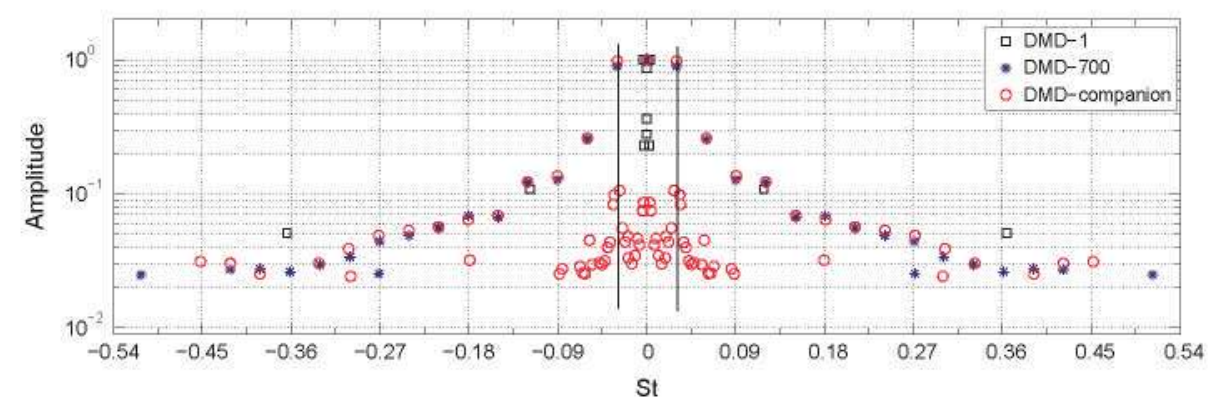

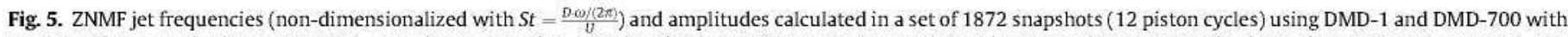

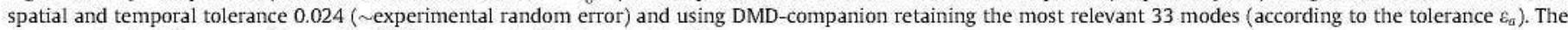
piston oscillation frequency $\left(f_{\text {exect }} \sim S t=0.03\right)$ is marked with black vertical lines.

the steady mode associated with mean flow $(S t=0)$ plus 11 harmonics and their complex conjugates. The accuracy of the frequency calculations is good, although somewhat worse than their DMD-700 counterparts considered above, since the relative errors defined above are now $E_{f}=0.38 \%$ for the fundamental frequency and $E_{R}=0.74 \%$ for the ratio of the 11 th harmonic to the first harmonic. Additionally, there is a second set of 21 pairs of complex conjugate modes that are irregularly clustered in a trapezoidalshaped region below the former set of modes. These additional modes are spurious in spite of the fact that they show a relatively high amplitude $\sim 0.1$, meaning that they could be wrongly considered as associated with transitional artifacts anticipating the turbulent flow in the far field. The fact that these modes are spurious is clearly seen in the reconstructions considered in the next subsection (see Fig. 6), which show several unphysical artifacts that are not present in the original data. Note that the DMD-700 computations do not show these unphysical artifacts. In other words, both the spatial cleaning carried out by HOSVD algorithm and the performance of DMD-700 algorithm automatically remove the undetermined modes (identified as spurious artifacts).

\subsection{DMD reconstructions}

A good way to check the accuracy and veracity of the approximation of the flow field as an expansion of DMD modes, is to perform the reconstruction of the original data. A more complete analysis of the reconstructions will be considered in the next section by using the more efficient iterative DMD- $d$ algorithm. Here, we focus on the two snapshots (one from the injection phase and another from suction phase) considered in Fig. 6. These snapshots are representative of the ZNMF topology patterns because the pseudo-streamlines show the vortex ring already identified in [41]. Fig. 6 shows the original data, the phase-averaged velocity fields, and the reconstruction obtained using all modes presented in Fig. 5, for each method. The original data and the DMDcompanion reconstruction are both quite noisy, whereas the phase averaged flow field and, especially, the DMD-700 reconstructions are cleaner and thus suitable to study the flow physics in more detail. Comparing the original data with the calculations in Fig. 6, the maximum and RMS errors are both smaller in the injection than in the suction phase (showing the larger complexity of this phase) and, for both the injection and suction phases, these errors are larger using the DMD-companion algorithm than using the other methods, showing their better performance of these methods. Specifically, the maximum and RMS errors, represented by MaxE and RMSE, respectively, are defined as

Error $=\frac{\left\|U-U_{\text {approx }}\right\|_{p}}{\|U\|_{p}}$ where $\|\cdot\|_{p}$ represents the $\mathrm{p}$-norm with $p=\infty$ and $p=2$ for the maximum and RMS errors, respectively, and $U, U_{\text {approx }}$ are the velocity in the original data and its reconstruction. This yields to the following,

- Phase-averaging: MaxE $\sim 1.71 \cdot 10^{-2}$ and RMSE $\sim 1.10 \cdot 10^{-3}$ (injection), and MaxE $\sim 3.97 \cdot 10^{-2}$ and RMSE $\sim 9.28 \cdot 10^{-4}$ (suction).

- DMD-700: MaxE $\sim 1.56 \cdot 10^{-2}$ and RMSE $\sim 1.14 \cdot 10^{-3}$ (injection), and MaxE $\sim 3.94 \cdot 10^{-2}$ and RMSE $\sim 8.81 \cdot 10^{-4}$ (suction).

- DMD-companion: MaxE $\sim 2.05 \cdot 10^{-2}$ and RMSE $\sim 2.6 \cdot 10^{-3}$ (injection), and MaxE $\sim 4.6 \cdot 10^{-2}$ and RMSE $\sim 2.18 \cdot 10^{-3}$ (suction).

Moreover, the DMD-700 reconstruction is topologically equivalent to the original data, while the DMD-companion reconstruction is not. This conclusion is further illustrated in Fig. 7, which shows in detail the streamwise velocity contours for the snapshots considered in Fig. 6. Obviously, the advantage of DMD- $d$ against phase-averaged technique is that the latter method is only capable of filtering out noise fluctuations whose mean value is zero, while DMD- $d$ detects and filters more general, systematic inconsistent errors according to a certain tolerance criterion. Thus, the number of snapshots that are required by DMD- $d$ is smaller than that required by phase-averaging. For this reason, despite phaseaveraging is applied in the entire set of data (20 cycles), DMD-d presents smoother and more precise results in a smaller data set (only 12 piston cycles).

In conclusion, the multidimensional DMD- $d$ algorithm summarized in Section 3.2 highly outperforms the standard DMD algorithms mentioned in Section 2.1, in connection with both the identified frequencies and the DMD reconstructions. However, even this method can be improved further by using its iterative version described in Section 3.3, which is applied to the ZNMF jet in the next section.

\section{Flow structures in the ZNMF jet using iterative HODMD}

Now that the advantages of the multidimensional DMD- $d$ algorithm have been illustrated, this algorithm is now further improved by applying it iteratively, as explained in Section 3.3.

The iterative multidimensional DMD- $d$ algorithm, with $d=700$ as in the last section, has been applied to the same experimental data set presented in previous section (1872 snapshots). Two different test cases have been investigated. In the first test case, the value of the spatial and temporal tolerances is similar to the value of the random estimated error of the experiment, $\varepsilon_{s}=\varepsilon_{t}=\varepsilon_{a}=0.024$ (as in the previous section), while in the sec- 

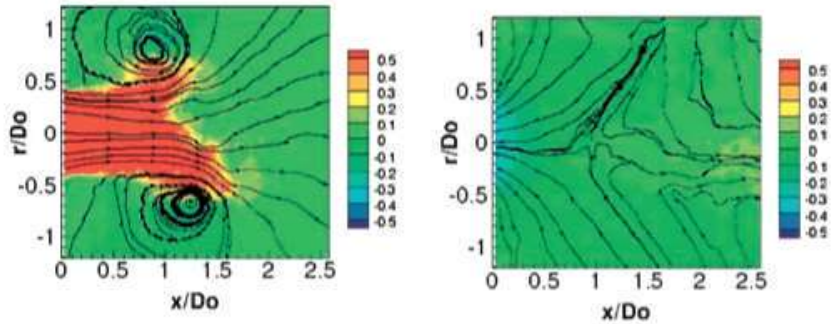

Original experimental data
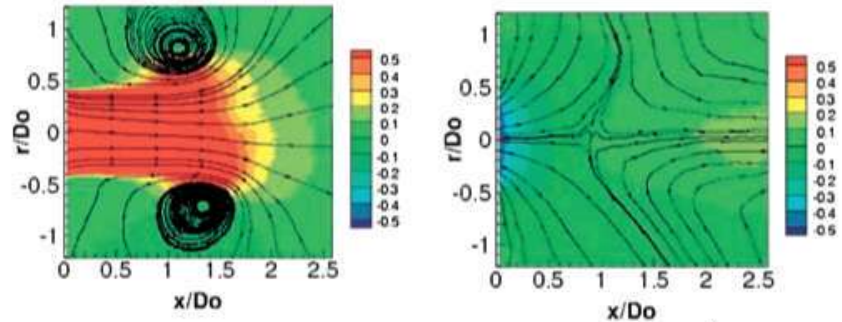

Phase-averaged velocity field with 3120 snapshots (20 piston cycles)
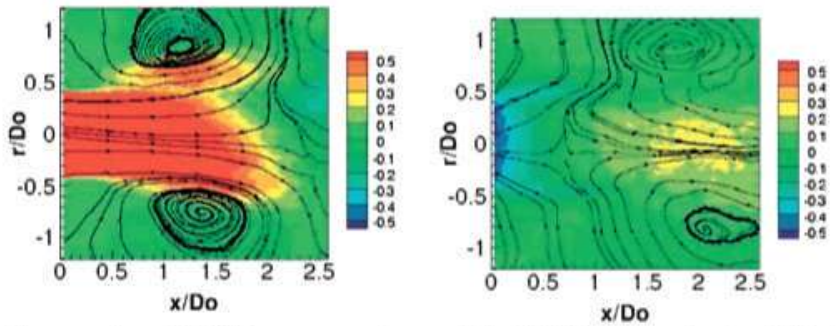

Reconstruction using DMD-companion with 1872 snapshots (12 piston cycles)
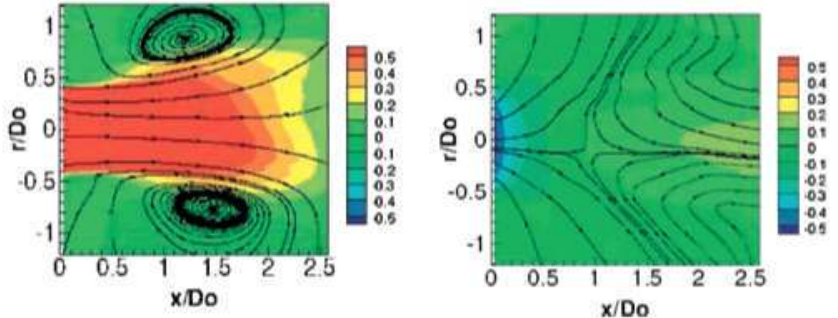

Reconstruction using DMD-700 with 1872 snapshots (12 piston cycles)

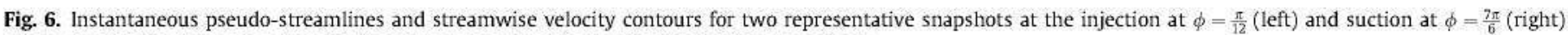
phase, considering both the original data and the reconstructions using the indicated methods.

ond case, these tolerances have been decreased to $\varepsilon_{s}=\varepsilon_{t}=\varepsilon_{a}=0.01$ ( $1 \%$ error). Using tolerances larger than 0.024 would reduce the number of modes retained by the method and consequently, would limit its good performance because the tolerance sets the smallest retained mode amplitude value. Fig. 8 compares the frequencies and amplitudes obtained in the two considered test cases. As can be seen, DMD- $d$ captures in both cases the steady mode, the fundamental frequency $S t=0.03$, and a set of harmonics of this frequency. However, in the case with smaller tolerance, the method captures 24 harmonics (and their complex conjugates) with relative errors $E_{f}=0.08 \%$ and $E_{R}=0.028 \%$ in the computation of the lowest and highest frequencies, respectively, while with the larger tolerances, the method captures 9 harmonics, with larger relative errors, namely $E_{f}=0.10 \%$ and $E_{R}=0.11 \%$. Table 3 shows the number of HOSVD modes in $r, x, t$ and the number of DMD- $d$ modes retained in each iteration in the studied test cases. As expected, the number of modes retained in the case with smaller tolerance is larger and, as also expected, the number of retained modes decreases along the iteration for both sets of tolerances. In particular, with the higher tolerance, the first iteration reflects the case considered in the previous section, which retains 33 DMD- $d$ modes. As anticipated, these modes correspond to the steady mode, 14 harmonics, and 2 pairs of complex conjugate spurious or low accuracy modes. In the second iteration, the number of HOSVD-spatial modes is reduced and, consequently, the number of DMD modes is also reduced to 23 , now with a single pair of complex conjugate mode for each frequency. Similarly, no spurious modes of this type are present in the remaining iterations.

The behavior of the method with the smaller tolerance is completely similar in connection with the identified frequencies, as Table 3 suggests. Namely, some of the 109 DMD modes retained in the first iteration are spurious, but these are removed in the subsequent iterations, at least in connection with the frequencies, which are identified very precisely, as explained above. Moreover, the fundamental frequency and its harmonics are reproduced with 

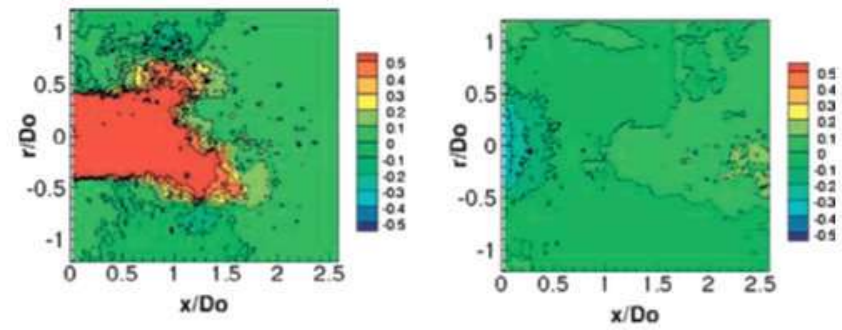

Original experimental data
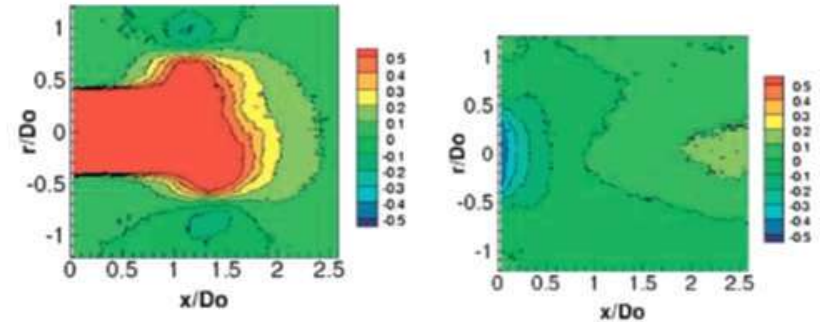

Phase-averaged velocity field with 3120 snapshots (20 piston cycles)
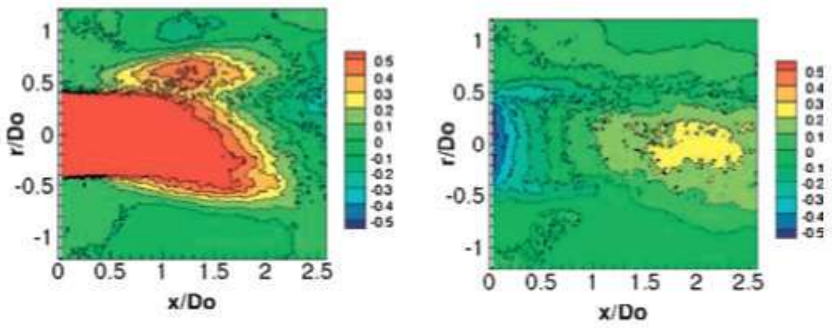

Reconstruction using DMD-companion with 1872 snapshots (12 piston cycles)
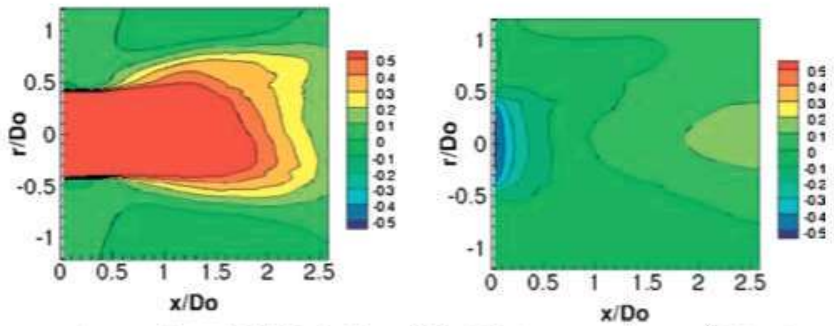

Reconstruction using DMD-700 with 1872 snapshots (12 piston cycles)

Fig. 7. More detailed plots of the streamwise velocity contours considered in Fig. 6.

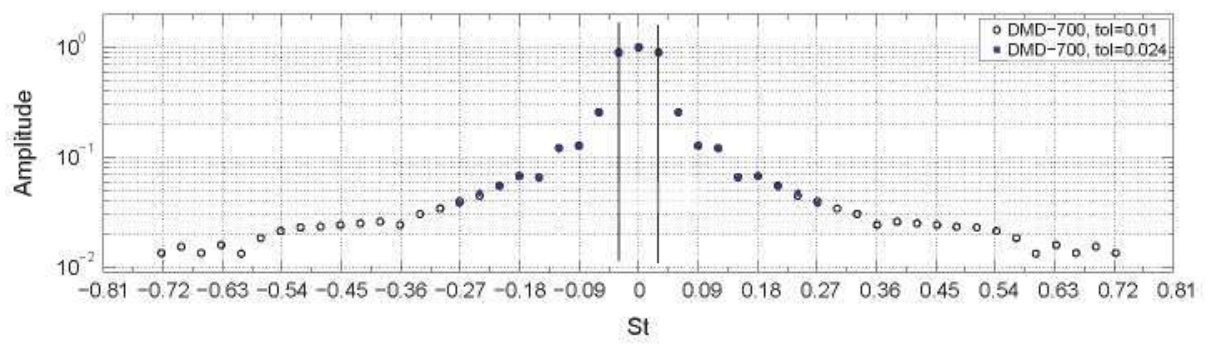

Fig. 8. Counterpart of Fig. 5 using the iterative multidimensional DMD-700 method with the indicated tolerances.

a better accuracy than with the larger tolerance, which is very promising because the smaller tolerance is smaller than the expected precision of the experimental measurements. However, a better description of the frequencies does not necessarily mean a better description of the flow structures. Thus, in the absence of a good reference (such as numerical simulations) for the physically meaningful features that should be expected in the ZNMF jet, it is not possible to assure that the features appearing in the additional DMD modes that are retained with the smaller tolerance are physically meaningful (namely, not due to spurious artifacts). Consequently, it will be the larger tolerance that will be considered in the remaining applications of the iterative DMD-700 method, in Figs. 9-11. 
Table 3

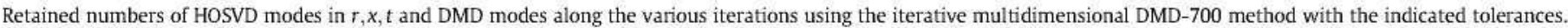

\begin{tabular}{|c|c|c|c|c|c|}
\hline Tolerance & Iteration & $r$ & $x$ & $t$ & \# of DMD- $d$ modes \\
\hline \multirow[t]{6}{*}{0.01} & 1 & 52 & 68 & 91 & 109 \\
\hline & 2 & 13 & 9 & 18 & 73 \\
\hline & 3 & 12 & 6 & 14 & 62 \\
\hline & 4 & 11 & 6 & 13 & 55 \\
\hline & 5 & 11 & 6 & 11 & 51 \\
\hline & 6 & 11 & 6 & 11 & 49 \\
\hline \multirow[t]{4}{*}{0.024} & 1 & 19 & 15 & 19 & 33 \\
\hline & 2 & 6 & 4 & 6 & 23 \\
\hline & 3 & 6 & 4 & 5 & 21 \\
\hline & 4 & 6 & 4 & 5 & 19 \\
\hline
\end{tabular}
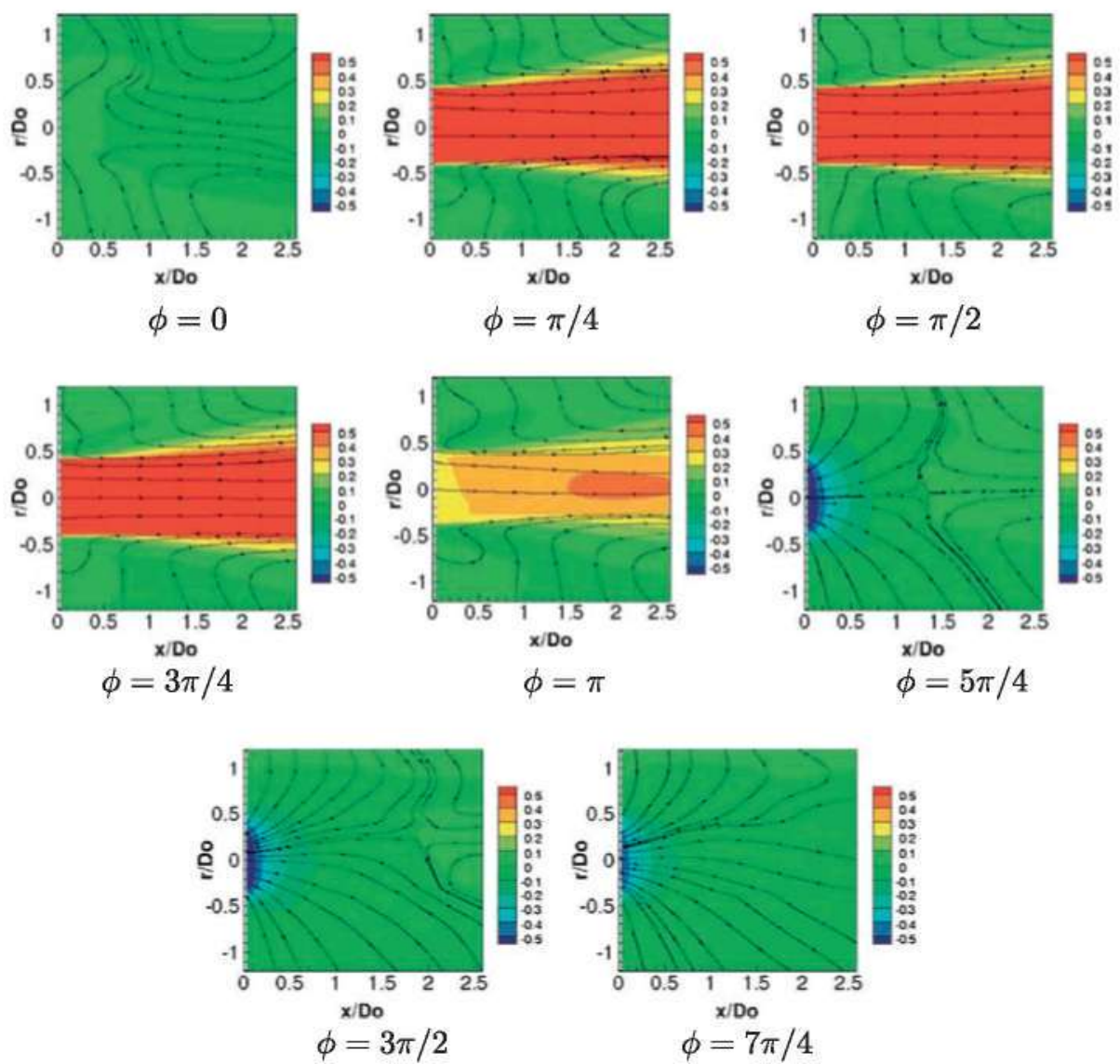

Fig. 9. Reconstruction of eight equispaced snapshots in the piston cycle, $0 \leqslant \phi<2 \pi$, retaining the modes indicated in Fig. 8 with the largest tolerances.

Fig. 9 shows a complete cycle of the piston movement. In the injection phase, $0<\phi<\pi$, the flow is impulsed through the jet orifice. After the initial transient, the instantaneous pseudostreamlines and streamwise velocity contours in this phase are similar to the patterns of a laminar continuous jet with radial entrainment. The first half of the injection phase, $0<\phi<\pi / 4$, is considered in more detail in Fig. 10. As can be seen, the sharp edge of the jet orifice causes the flow to separate and roll up forming a vortex ring that moves downstream due to its self-induced velocity. This is consistent with phase-averaged results presented in [41] (using 5 sets of 20 cycles, 100 snapshots, as mentioned in Section 4.2), with the exception of the first image, for $\phi=\pi / 36$, which shows a saddle point near the axis of the jet. The existence of this saddle point could be anticipated by topological arguments, since at the very beginning of the suction phase, the flow near the axis of the jet must move upstream near the orifice (due to suction from the orifice) but still moves downstream far from the orifice (as in the latter stages of the former suction stage). These opposite trends obviously produce an stagnation point somewhere near the axis of the jet (not necessarily on the axis of the jet because the flow is not strictly axisymmetric due to experimental imperfection). However, this saddle point is short-lived (namely, it moves downstream very fast) due to the high Reynolds number, which seemingly explains why it was not identified in the phase averaged pseudostreamlines considered in [41]. Note, on the other hand, that for very low Reynolds number, the flow is linear and inherits the spatio-temporal reflection symmetry of forcing, namely

$v_{x} \rightarrow-v_{x}, \quad t \rightarrow T / 2-t, \quad$ or $\phi \rightarrow \pi-\phi$.

Thus, in this case, the encountered saddle point in the injection phase is the image of the saddle point in the suction phase (considered below) under this reflection symmetry and would move with the same (opposite) velocity. In other words, at low Reynolds num- 

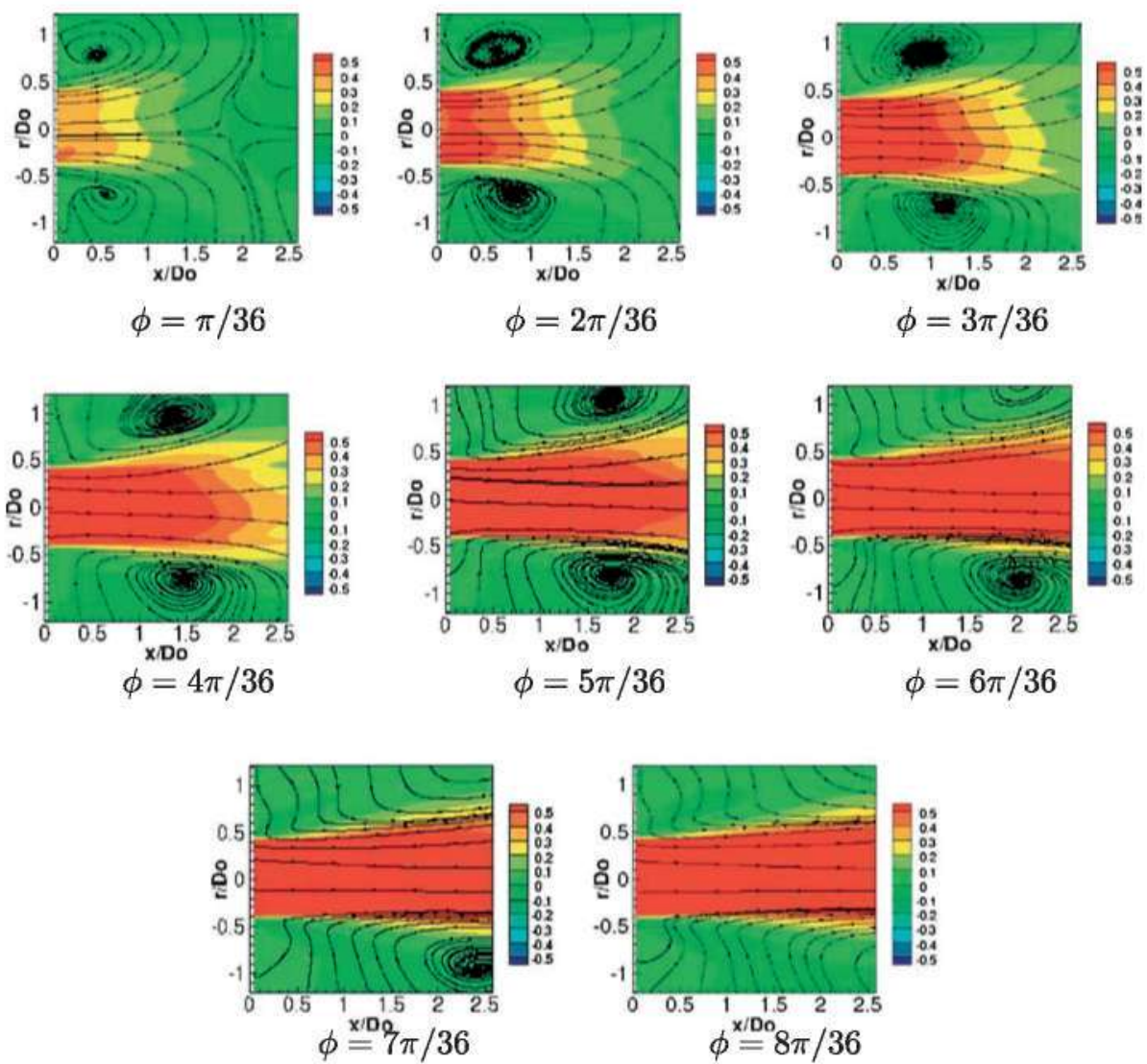

Fig. 10. Counterpart of Fig. 9 for eight equispaced snapshots in the first half of the injection cycle, $0<\phi<\pi / 4$.
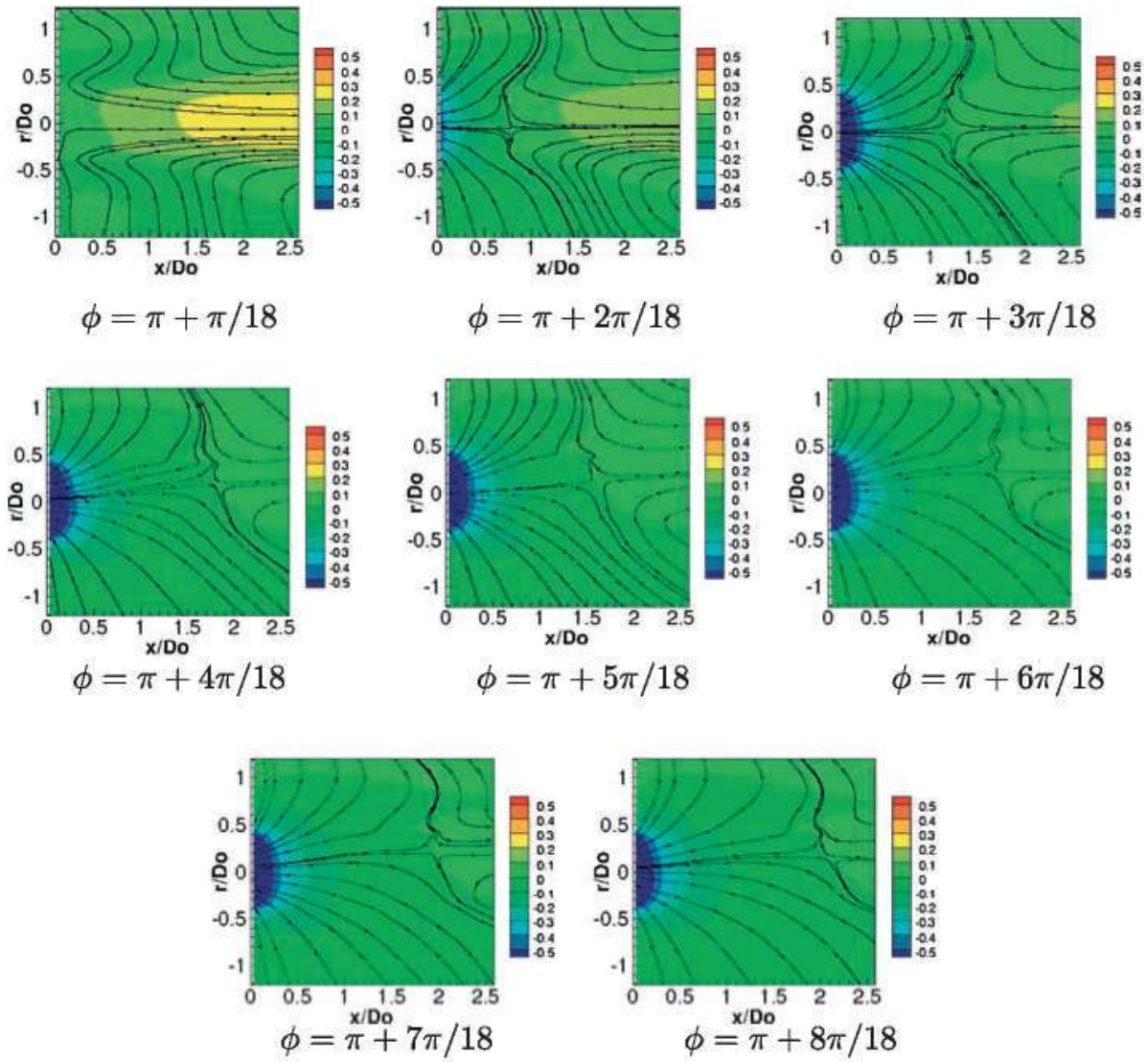

Fig. 11. Counterpart of Fig. 9 for eight equispaced snapshots in the last part of the injection cycle, $\pi<\phi<3 \pi / 2$. 
ber, the new saddle point in the injection phase is expected to last for a longer timespan.

Concerning the suction phase (for $\pi<\phi<2 \pi$ in Fig. 9), the pseudo-streamlines depict a saddle point that moves downstream from the jet orifice. Using a similar topological argument as that used above for the injection phase, it is obvious that, as more clearly seen in the more detailed description in Fig. 11, upstream of this saddle point the flow is sucked upstream through the jet orifice, while downstream of the saddle point the flow continues to move downstream, as it occurred in the former injection phase.

Summarizing the results presented in Figs. 9-11, the HODMD method, using only 12 piston cycles of the data set, perfectly reproduce the results in [41], which were obtained using a larger data set, namely 5 sets of 20 piston cycles. This fact sheds light on the possibility of using HODMD in experiments in which a smaller number of data are collected, due to limitations in the set up.

Let us now consider the 19 DMD modes (see Fig. 8 and Table 3) that were retained in the DMD expansion (5.1) to reconstruct the flow structures in Figs. 9-11. The two velocity components defined in (5.3) for these 19 modes are plotted in Figs. 12 and 13, considering separately the mean flow ( $S t=0$ ), which is real, and the real and imaginary parts for each pair of complex conjugate modes associated with the remaining 9 harmonics. Concerning the latter pairs of complex conjugate modes, according to the DMD expansion (5.1), the velocity components produced by the complex conjugate pair associated with the frequencies, $\omega_{m}$ and $-\omega_{m}$, can be written in terms of the real and imaginary parts of the modes $\boldsymbol{u}_{m}, \operatorname{Re}\left(\boldsymbol{u}_{m}\right)$ and $\Im\left(\boldsymbol{u}_{m}\right)$, respectively, as

$a_{m} \boldsymbol{u}^{m} \mathrm{e}^{\mathrm{i} \omega_{m} t_{k}}+$ c.c. $=2 a_{m}\left[\operatorname{Re}\left(\boldsymbol{u}_{m}\right) \cos \left(\omega_{m} t_{k}\right)-\Im\left(\boldsymbol{u}_{m}\right) \sin \left(\omega_{m} t_{k}\right)\right]$

Thus, this contribution reflects a monochromatic oscillation that subsequently visits the states $\pm 2 a_{m} \operatorname{Re}\left(\boldsymbol{u}_{m}\right)$ and $\mp 2 a_{m} \Im\left(\boldsymbol{u}_{m}\right)$ as time increases. The upper plots in Fig. 12 show the steady mode (only the real real parts of $u_{x}^{(1)}$ and $u_{r}^{(1)}$ because the mode is real), which corresponds to the mean flow. As seen, the streamwise velocity component is much larger than the radial component except near the orifice $(x=0)$ and the shape of the former component is similar to its counterpart in a continuous jet. Also, these plots show that the mean flow approximately exhibits an up-down reflection symmetry. The remaining plots in Fig. 12 and those in Fig. 13 show the DMD-700 modes associated with the fundamental frequency and the retained eight harmonics. The overall shape of the streamwise
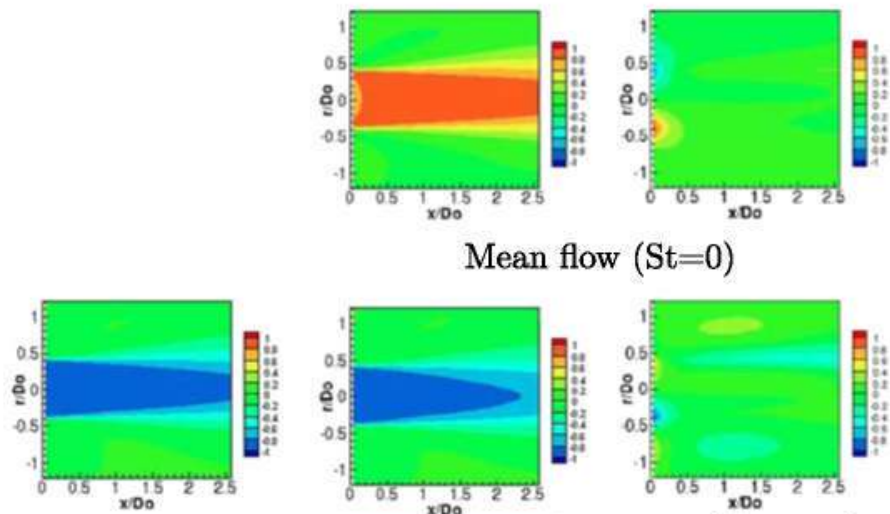

\section{Mean flow $(\mathrm{St}=0)$}
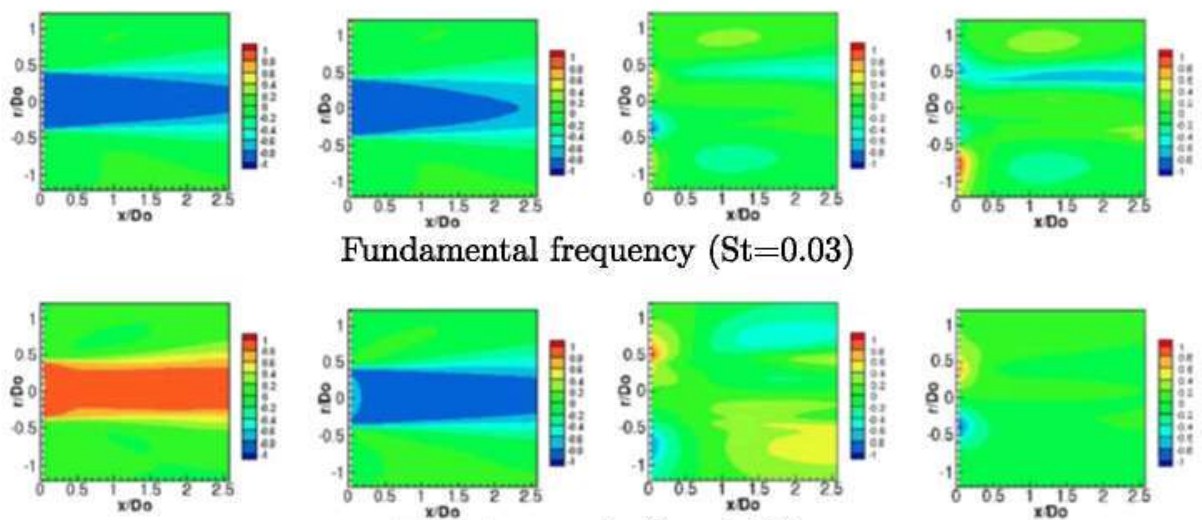

First harmonic $(\mathrm{St}=0.06)$
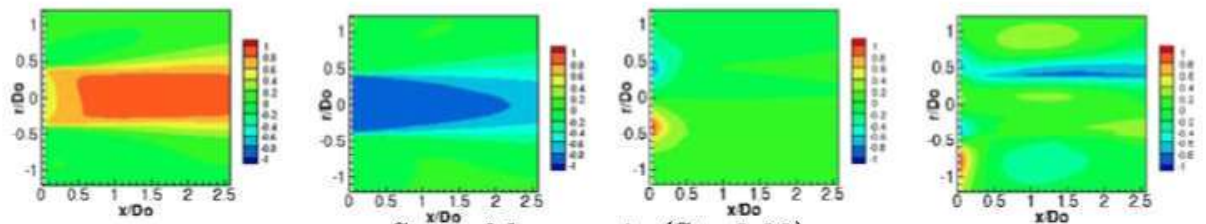

Second harmonic $(\mathrm{St}=0.09)$
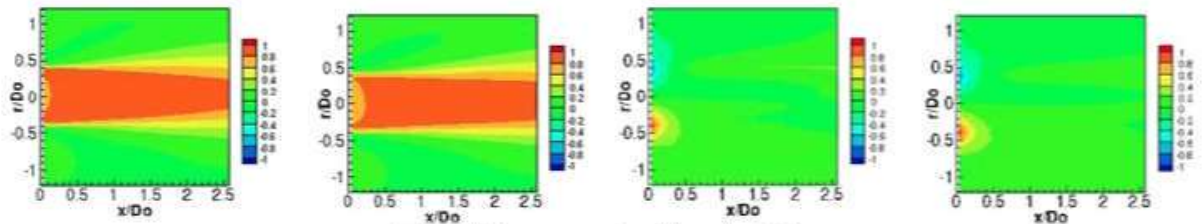

Third harmonic $(\mathrm{St}=0.12)$

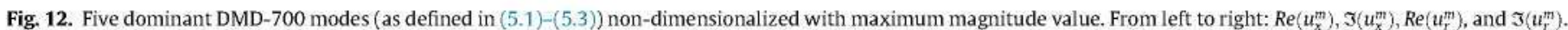


component of these oscillating modes is similar to the mean flow in all these cases, in the sense that the intensity is largest near the axis of the jet. However, the difference between these modes is important in order to understand its role in the time-dependent jet, which oscillates with the frequency of the piston and the frequencies of the retained harmonics. The interaction of the mean flow and these harmonics leads to the non-linear dynamics of the jet.

Considering Figs. 12 and 13 in more detail, it is possible to find some slight differences in the shape of the retained modes:

- The real and imaginary parts of the streamwise velocity component of the mode associated with the fundamental frequency, $u_{x}^{(2)}$, are both maximum near the orifice and progressively decay downstream, forming at $x / D_{0}=2$ a profile with a smooth Gaussian shape. Moreover, both the real and imaginary parts of $u_{x}^{(2)}$ do not differ much from each other, meaning that the associated reconstruction according to (6.2) correspond to an oscillation that is approximately in-phase. However, the small difference between the real and imaginary parts of $u_{x}^{(2)}$ promote an outof-phase oscillation, which is to be expected because the jet patterns (vortices and saddles) travel in the streamwise direction.
On the other hand, the real and imaginary parts of the radial component of this mode, $u_{r}^{(2)}$, are both non-symmetric, which could be associated with a symmetry breaking of the jet produced by an azimuthal instability of this mode. The resulting azimuthal modes could be related to the vortices breaking down and their subsequent transition to turbulent flow [36].

- Concerning the remaining modes, for $m=3, \ldots, 10$, the real and imaginary parts of the streamwise velocity components $u_{x}^{m}$ generally peak at different locations, and also vary from one harmonic to another. Invoking (6.2), this means that these harmonics produce oscillations that are clearly out-of-phase, and fairly different from one harmonic to another. As for the radial component of the modes, $u_{r}^{m}$, both their real and imaginary parts generally peak near the orifice, which is consistent with the formation of the vortex rings in this region. However, consistently with the shape of the streamwise velocity component (note that $u_{x}^{m}$ and $u_{r}^{m}$ must satisfy the continuity equation), the radial components must show local peaks at different locations of the domain, which are appreciated only in some of the modes due to the color scale. As above, the interactions of these out-of-phase oscillations are needed to explain the formation
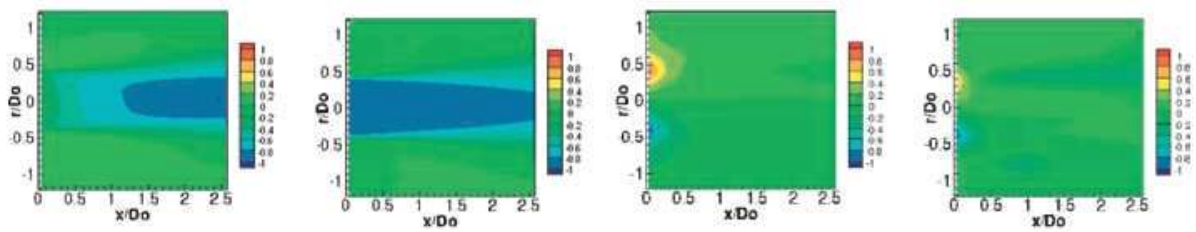

Fourth harmonic $(\mathrm{St}=0.15)$
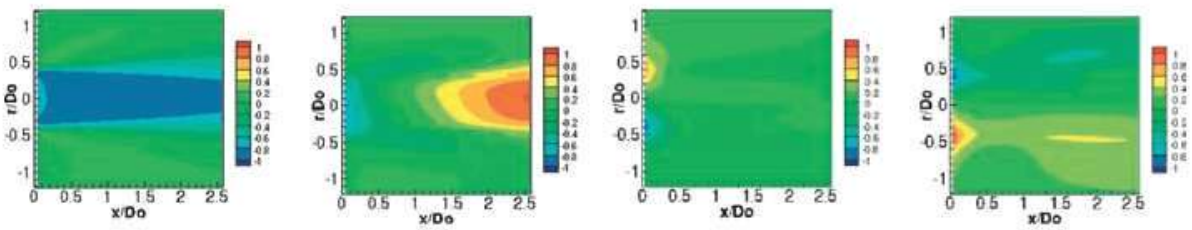

Fifth harmonic $(\mathrm{St}=0.18)$
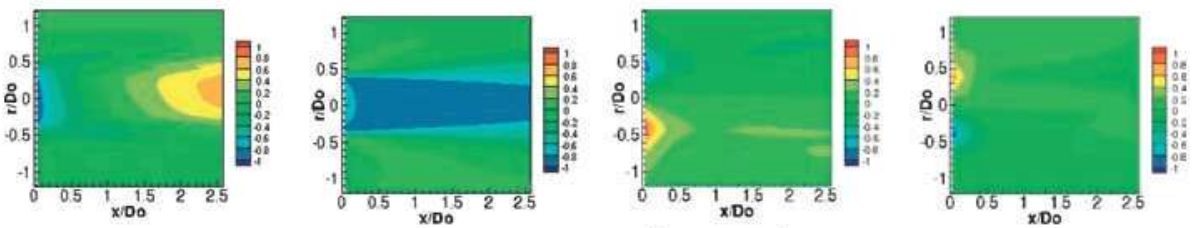

Sixth harmonic $(\mathrm{St}=0.21)$
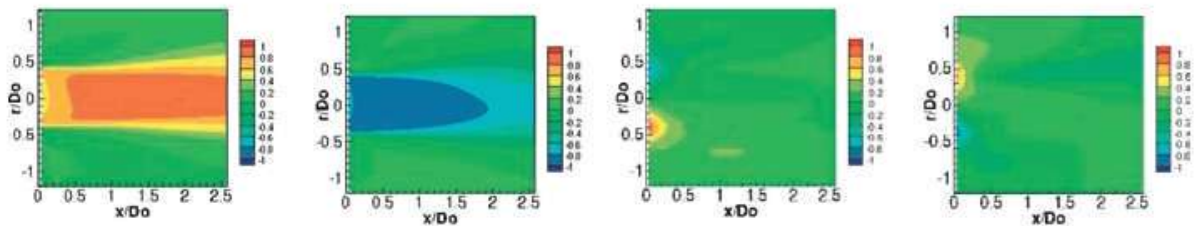

Seventh harmonic $(\mathrm{St}=0.24)$
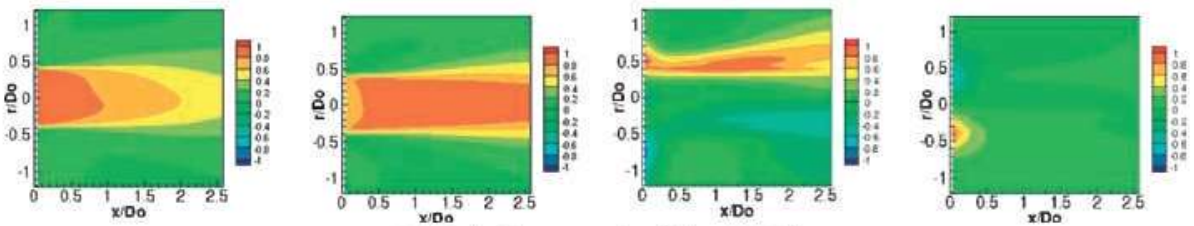

Eighth harmonic $(\mathrm{St}=0.27)$

Fig. 13. Counterpart of Fig. 12 for the 4 th- 8 th harmonics of the fundamental frequency. 

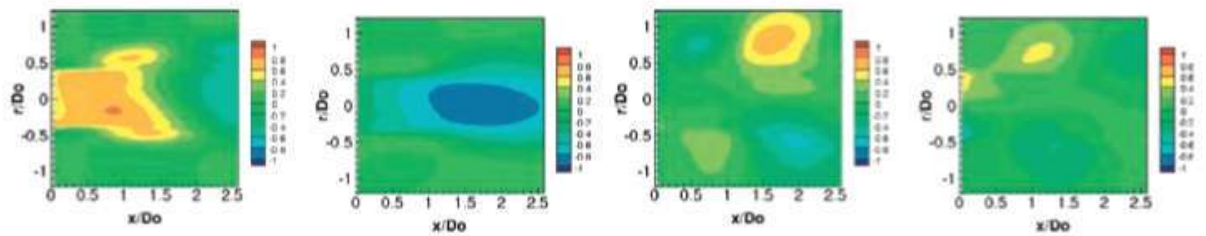

Thirteenth harmonic $(\mathrm{St}=0.42)$
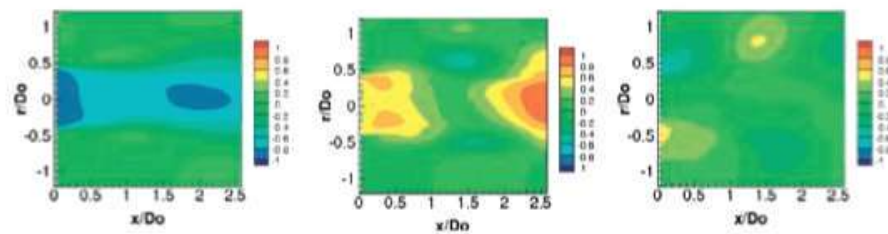

Eighteenth harmonic $(\mathrm{St}=0.57)$

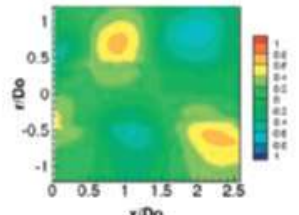

Fig. 14. Counterpart of Fig. 12 for the 13 th and 18 th harmonics of the fundamental frequency.

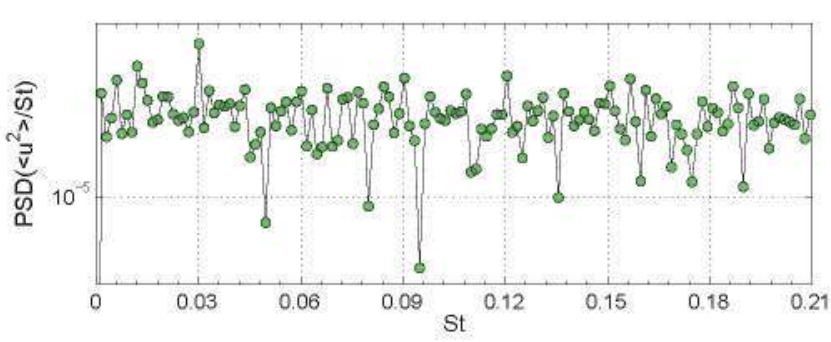

Fig. 15. PSD applied on a representative point of the computational domain $\left(\left(x / D_{0}, r / D_{0}\right) \in(0.5,0.4)\right)$.

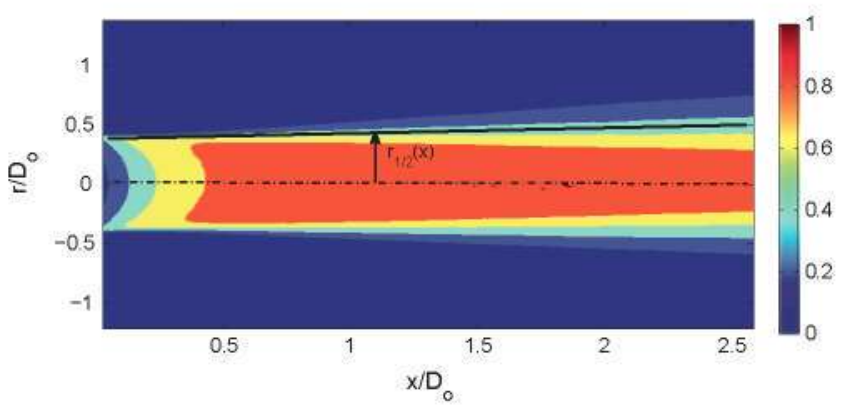

Fig. 16. Mean streamwise velocity using 20 piston cycles. Black line defines the half width of mean axial velocity profile of the ZNMF jet $r_{1 / 2}(x)$.

and streamwise motion of the vortices and saddles that were described above in this section.

As a final comment, it is somewhat striking how the modes plotted in Figs. 12 and 13, whose structure is somewhat simple, organize themselves though the DMD expansion (5.1) to produce the fairly complex spatio-temporal structures depicted in Figs. 911. For this reason, the spatial scale of the modes is maintined mostly constant at this relatively low frequencies. These 9 harmonics represent large scale (and complex) structures such as the short-lived vortex ring or the saddle point found in the injection and suction phases of the piston, respectively. Smaller scales are related to still larger frequencies. For illustration, Fig. 14 shows the 13th and 18th harmonics of the fundamental frequency (obtained with the tolerance 0.01 , smaller than the experimental data uncertainty). These modes show how the very high frequency modes promote very small spatial scales.
Finally, let us mention that, as could be guessed from Fig. 5, the DMD-companion algorithm is also able to compute the highest amplitude modes, with a shape that is qualitatively similar to that presented above, but much noisier. The reason is that in DMDcompanion algorithm, it is not possible to perform the dimension reduction step of SVD that cleans the data. The multidimensional HODMD method, instead, provides much better results, since it is capable to: (i) clean noisy data using a reduced number of snapshots, (ii) avoid spurious modes and provide accurate and reliable results, and (iii) calculate low amplitude modes (under experimental uncertainty levels) related to small spatial amplitudes.

\section{HODMD and spatial growth rates}

The analysis previously presented is a global analysis calculated in time (applied to data equispaced in time) and gives a set of spatio-temporal data as an expansion of spatial modes that oscillate in time with a certain frequency (Eq. (1.1)) in the entire domain. The experimental data are collected once the transient stage is stabilized, meaning that they are saturated in time (not growing or decaying dynamics). Consequently, when HODMD is applied to such data set, the calculated growth rates turn out to be zero (within the accuracy in the computations).

Nevertheles, transition to turbulence in the far field of the ZNMF jet can be studied by identifying spatially developing instabilities in the near field. On the one hand, these instabilities can be calculated as proposed by Schmid [32], using DMD to analyze a set of data equispaced in space. In a similar way, it is possible to use HODMD to perform the same analysis. Thus, in the snapshot matrix defined in (2.2), each snapshot $\boldsymbol{v}_{k}$ would be equispaced in space instead of time. On the other hand, these instabilities are usually identified in the literature by computing the PSD (or FFT) spectrum at various representative points of the spatial domain and comparing the amplitude evolution of the dominant frequencies. In other words, spatially growing instabilities are related to the spatial increase of the amplitude of the dominant frequencies [23], which may be identified via a local analysis. In order to show the good performance of HODMD algorithm, this second method has been used to identify the spatially growing instabilities.

Fig. 15 shows the temporal PSD spectrum calculated on a representative point of the computational domain using the same data set used above in HODMD computations. As can be seen, the PSD spectrum is fairly noisy and barely allows for computing noting but the fundamental frequency, at $S t=0.03$. PSD uncertainty is reduced by using a larger set of data, but this might be a problem in cases in which the data collected are limited. 


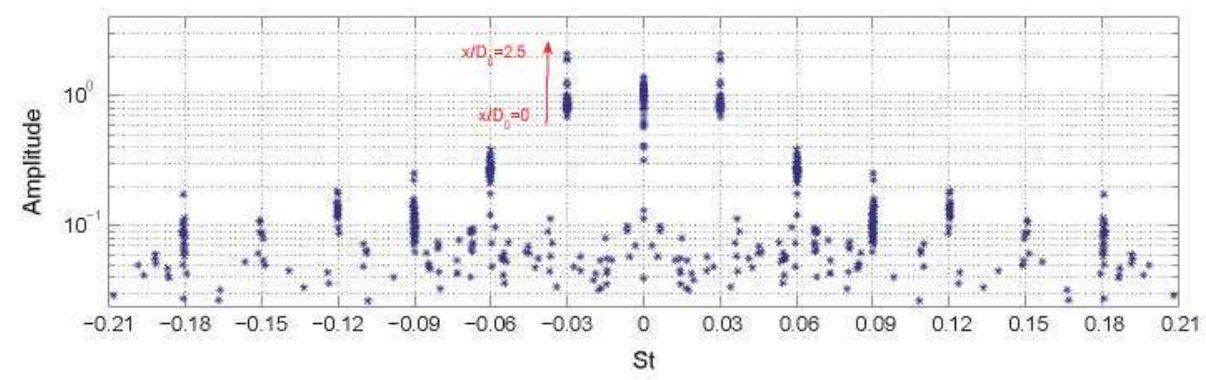

Fig. 17. Counterpart of Fig. 5 using HODMD in 75 points defining the line $r_{1 / 2}(x)$.

The good performance of HODMD makes this tool a suitable alternative to PSD analysis, which provides accurate results using a smaller number of snapshots (or points) [26]. Therefore, spatially developing instabilities will be identified below by applying this technique at various representative points of the domain. Since the main goal is to identify the driven mechanism that produces transition to turbulence in the far field, several points have been taken along a line, denoted as $r_{1 / 2}(x)$, in the streamwise direction. As indicated in Fig. 16, the $r_{1 / 2}(x)$ line is defined, at each streamline position, by the radius where the mean axial velocity has decayed by half from its centerline value, which is seen as representative in both the injection and suction phases, following the vortex ring and saddle point trajectories [41].

A set of 75 equispaced points have been taken along the $r_{1 / 2}(x)$ line and HODMD has been performed at each one of these points independently, with the same parameters as in the previous section: 1872 equispaced values of time, DMD-700, and $\varepsilon_{t}=\varepsilon_{a}=0.024$. The analysis has been performed using both the streamwise and normal velocity components. For simplicity, neither the iterative process nor HOSVD has been used. In fact, the iterative process would help to remove spurious artifacts, improving slightly the results presented below, by totally removing spurious modes. On the other hand, since HODMD is applied independently at each of the 75 points selected above, it has no meaning separating into spatial directions, which is the main purpose of using HOSVD. Additionally, this fact also explains that standard DMD would not be capable to properly analyze these data because, at each point, the spectral complexity $M$ is very large (let us remember that in previous section it was shown that the flow analyzed is periodic, retaining a large number of harmonics). Thus, $M$ is expected to be much larger than the spatial complexity, which is $N=2$ (because the two velocity components are used).

The amplitudes vs. frequencies resulting from applying HODMD at the selected 75 points is given in Fig. 17, where it is seen that (as in the previous sections) the dominant amplitude is associated with the forcing frequency, $S t=0.03$. Ignoring those spurious points in the lower part of the plot, those points in the upper part of the vertical line at $S t=0.03$ indicate the dominant amplitudes along the 75 selected points. This amplitude increases with the streamwise spatial coordinate $x$ about an order of magnitude $\left(\log \left(a_{m} / a_{0}\right) \simeq 1.2\right)$ in the interval $0 \leqslant x / D_{0} \leq 2.5$, which suggests the transitional character of this flow, which is thus expected to be turbulent in the far field (i.e.: $x / D_{0} \simeq 20$ ). For the remaining harmonics, the spatial growth rate is similar, though it is even somewhat larger for the second harmonic $(S t=0.09)$. This behavior is produced by the interaction of the DMD modes presented in previous section, which is thus responsible for the transition to turbulence process that takes place in the far field.

\section{Concluding remarks}

A new method has been presented to study flow structures in highly complex fluid flows. The method combines the HODMD algorithm (DMD-d) presented in [26] with a HOSVD algorithm that is applied first in order to both cleaning noisy data and to avoid spatial redundancies. The algorithm has been applied to a set of PIV experimental data (using a small number of snapshots) with the aim at studying the flow structures in the near field of a ZNMF jet. The flow conditions under which the experiment was carried out led to a fully turbulent jet in the far field. Both, the high complexity of the flow and the noise added in the experiment, made this case suitable to test the performance of this new method.

Since the ZNMF jet is periodic, the temporal growth rates of the DMD modes vanish (to the accuracy of the computations). The results obtained, DMD- $d$ modes and frequencies, were used to reconstruct the instantaneous original data provided by the experiment as a Fourier-like expansion in DMD modes. These results were compared with the original instantaneous field, with the phase-averaged field (calculated in this article and in the literature [41] taken a much larger amount of data), and with results obtained with standard DMD [32]. This comparison puts in evidence both the superior performance of the method developed here and its capability to clean highly complex noisy data. This fact permits discriminating spurious modes from the real flow dynamics. This is useful, on the one hand, to provide an accurate description of the instantaneous flow field and, on the other hand, to prevent from misleading interpretations of spurious artifacts as responsible for transition to turbulence process.

Taking advantage of the synergies between the HOSVD and HODMD steps of the algorithm, the method itself was applied iteratively over the given data set. The resulting iterative method progressively smooths and cleans the noisy data along the iterations, and the final reconstruction describes the main flow features associated to the injection and suction phases typical of the ZNMF jet quite well. These include the origin and evolution of the vortex ring in the injection phase and the formation and propagation of a well known saddle point generated close to the jet exit orifice in the suction phase, which divides the flow into a region sucked by the orifice and a region where the flow continues moving downstream. In addition, a saddle point was identified at the beginning of the injection phase that does not seem to have been previously mentioned. Using a relative tolerance comparable to the expected experimental precision, the iterative method was capable of capturing up to 19 DMD modes. These modes corresponded to the mean flow and 9 harmonics (plus their complex conjugates), with fundamental frequency equal to the ZNMF jet driven frequency. Studying in some detail these DMD spatial modes, some of the spatio-temporal flow structures were identified and related to 
the presence of secondary vortical structures seemingly due to an azimuthal instability and the vortex breakdown previously explained in the literature [36]. Therefore, from this detailed analysis, it is possible to suggest that an azimuthal instability could be the main mechanism that produces the transition to turbulence in the far field from this flow. Additionally, the spatial scale of the DMD modes captured is large, since they represent complex spatio-temporal structures (a short-lived vortex and a saddle point in the injection and suction phases, respectively) and it is necessary to retain higher frequency harmonics to reproduce smaller scales.

Finally, HODMD has been used as an alternative to PSD to accurately identify spatially growing instabilities, using a reduced number of snapshots. It was found that the amplitude of the harmonics previously identified in the temporal analysis grows more than an order of magnitude in the region studied and, consequently, can be seen as responsible to the transition to turbulence process carried out in the far field.

\section{Acknowledgments}

This work was partially supported by the Spanish Ministry of Economy and Competitiveness, under grants TRA2013-45808-R and TRA2016-75075-R. The support of the ARC via a Discovery Grant are gratefully acknowledged. The authors are indebted to the anonymous referees for some useful comments that helped to improve presentation of the results.

\section{References}

[1] R.A. Antonia, Conditional sampling in turbulence measurements, Ann. Rev. Fluid Mech. 13 (1981) 131-156.

[2] S. Bagheri, Koopman-mode decomposition of the cylinder wake, J. Fluid Mech. 726 (2013) 596-623.

[3] N. Benito, 1. Arias, A. Velazquez, J.M. Vega, Real time performance improvement of engineering control units via higher order singular value decomposition: application to a SI engine, Control Eng. Prac. 19 (11) (2011) $1315-1327$.

[4] S.L. Brunton, J.L. Proctor, J.N. Kutz, Discovering governing equations from data: sparse identification of nonlinear dynamical systems, PNAS 113 (2016) 39323937.

[5] J.E. Carter, J. Soria, The evolution of round zeron-net-mass-flux jets, J. Fluid Mech. 472 (2002) 167-200.

[7] L.N. Cattafesta, M. Sheplak, Actuators for active flow control, Annu. Rev. Fluid Mech. 43 (2010) 247-272.

[8] K.K. Chen, J.H. Tu, C.W. Rowley, Variants of dynamic mode decomposition: boundary condition, Koopman and Fourier analyses, J. Nonlinear Sci. 22 (2012) 8871-8875.

[9] L De Lathawer, B. De Moor, J. Vandewalle, On the best rank-1 and rank$\left(R_{1}, R_{2}, \ldots, R_{N}\right)$ approximation of higher-order tensors, SIAM J. Matrix. Anal. Appl. 21 (2000) 1324-1342.

[10] L. De Lathawer, B. De Moor, J. Vandewalle, A multilinear singular value decomposition, SIAM J. Matrix. Anal. Appl. 21 (2000) 1253-1278.

[11] S. de Lucas, J.M. Vega, A. Velazquez, Aeronautic conceptual design optimization method based on higher order singular value decomposition, AIAA J. 49 (2011) 2713-2725.

[12] E. DeMont, J. Gosline, Mechanics of jet propulsion in the hydromedusan jellyfish, polyorchis penicillatus, J. Exp. Biol. 134 (1988) 347-361.

[13] D. Duke, J. Soria, D. Honnery. An error analysis of the dymamic mode decomposition, Exp. Fluids 52 (2) (2012) 529-542.

[14] E. Ferrer, J. de Vicente, E. Valero, Low cost 3D global instability analysis and flow sensitivity based on dynamic mode decomposition and high-order numerical tools, Int J. Num. Meth. Fluids 76 (3) (2014) 169-184.
[15] A. Fouras, ]. Soria, Accuracy of out-of-plane vorticity measurements using inplane velocity vector field data, Exp. Fluids 25 (1998) 409-430.

[16] A. Glezer, M. Amitay, Synthetic jets Annu, Rev. Fluid Mech. 34 (2002) 503-529.

[17] F. Gómez, S. Le Clainche, P. Paredes, M. Hermanns, V. Theofilis, Four decades of studying global linear instability: progress and challenges, AlAA J. 50 (12) (2012) 2731-2743.

[18] A. González-Espinosa, N. Buchmannb, A. Lozanoa, J. Soria, Time-resolved stereo PIV measurements in the far-field of a turbulent zero-net-mass-flux jet Exp, Therm. Fluid Sci. 57 (2014) 111-120.

[19] G. Haller, An objective definition of a vortex, J. Fluid Mech. 525 (2005) 1-26.

[20] J.C.R. Hunt, A. Wray, P. Moin, Eddies, stream, and convergence zones in turbulent flows, Center for Turbulence Research Report, CTR-S88, 1988.

[22] B. Koopman, Hamiltonian systems and transformations in Hilbert space, Proc. Natl Acad. Sci. USA 17 (1931) 315-318.

[23] S. Le Clainche, I. Li, V. Theofilis, J. Soria, Time-resolved Particle Image Velocimetry and structural analysis of a hemisphere-cylinder at low Reynolds number and large angle of incidence AIAA 2012-3275, 2012. doi: http://dx.doi.org/10.2514/6.2012-3275.

[24] S. Le Clainche, I.I. Li, V. Theofilis, J. Soria, Flow around a hemisphere-cylinder at high angle of attack and low Reynolds number. Part I: Experimental and numerical investigation, Aerospace Sci. Technol. (2015) 77-87, http://dx.doi. org/10.1016/j.ast.2014.03.017 44.

[25] S. Le Clainche, D. Rodríguez, V. Theofilis, J. Soria, Flow around a hemispherecylinder at high angle of attack and low Reynolds number. Part II: POD and DMD applied to reduced domains, Aerospace Sci. Technol. (2015) 88-100, http://dx.dol.org/10.1016/j.ast.2014.10.009 44.

[26] S. Le Clainche, J.M. Vega, Higher order dynamic mode decomposition, SIAM ]. Appl. Dyn. Syst. 16 (2) (2017) 882-925.

[27] L.S. Lorente, J.M. Vega, A. Velazquez, Generation of aerodynamics databases using high-order singular value decomposition, J. Aircraft 45 (2008) 1779 1788.

[28] L.S. Lorente, J.M. Vega, A. Velazquez, Compression of aerodynamic databases using high-order singular value decomposition, Aerospace Sci. Technol. 14 (2010) 168-177.

[29] A. Pavlova, M. Amitay, Electronic cooling using synthetic jet impingement, J. Heat Transfer 128 (2006) 897-907.

[30] W.H. Press, S.A. Teukolsky, W.T. Vetterling, B.P. Flannery, Numerical Recipes in C, Cambridge University Press, 1992.

[31] C.W. Rowley, I. Mezic, S. Bagheri, P. Schlatter, D.S. Herningson, Spectral analysis of nonlinear flows, J. Fluid Mech. 641 (2009) 115-127.

[32] P.J. Schmid, Dynamic mode decomposition of numerical and experimental data, J. Fluid Mech. 656 (2010) 5-28.

[33] P.]. Schmid, Applications of the dynamic mode decomposition to experimental data, Exp. Fluids 50 (2011) 11231-71130.

[34] P.]. Schmid, L. Li, M.P. Juniper, O. Pust, Applications of the dynamic mode decomposition, Theor. Comp. Fluid Dyn. 25 (2011) 2491-7259.

[35] P.]. Schmid, D. Violato, F. Scarano, Decomposition of time-resolved tomographic PIV, Exp. Fluids 52 (2012) 1567-1779.

[36] B.L. Smith, A. Glezer, The formation and evolution of synthetic jets, Phys. Fluids 10 (9) (1998) 2281-2297.

[37] B.L. Smith, A. Glezer, Jet vectoring using synthetic jets, J. Fluid Mech. 458 (2002) $1-24$.

[38] L. Sirovich, Turbulence and the dynamic of coherent structures, parts 1-III, Q. Appl. Math. 45 (3) (1987) 561.

[39] J. Soria, An investigation of the near wake of a circular cylinder using a videobased digital cross-correlation particle image velocimetry technique, Exp. Therm. Fluid Sci. 12 (1996) 221-233.

[40] J. Soria, T.h. New, T.T. Lim, K. Parker, Multigrid CCDPIV measurements of accelerated flow past an airfoil at an angle of attack of 30, Exp. Therm. Fluid Sci. 27 (2003) 667-676.

[41] 」. Soria, Experimental studies of the near-field: spatio-temporal evolution of zero-net-mass-flux (ZNMF) jets, Vortex Rings and Jets: Fluid Mechanics and Its Applications, vol. 111, Springer, 2015, pp. 61-92.

[42] F. Takens, Detecting strange attractors in turbulence, in: D.A. Rand, L-S. Young (Eds.), Lecture Notes in Mathematics, Springer-Verlag, 1981, pp. 366-381.

[43] L.R. Tucker, Some mathematical notes on three-mode factor analysis, Psikometrica 31 (1996) 279-311.

[44] H. Wang, S. Menon, Fuel-air mixing enhancement by synthetic microjets, AIAA J. 39 (2001) 2308-2319.

[45] S.E. Widnall, C. Tsai, The instability of a thin vortex ring of constant vorticity, Proc. R. Soc. Lond., A 267 (1977) 273-305. 